Asia Pac. J. Math. 2020 7:3

$1-15$

ASIA PACIFIC ACADEMIC

\title{
MATHEMATICAL MODEL OF THE TRANSMISSION DYNAMICS OF DENGUE FEVER IN TWO CATEGORIES WITH VACCINATION AND MIGRATION EFFECT
}

\author{
R. N. UCHE-IWE, Z. C. OPARA, S. C. INYAMA*, A. OMAME \\ Department of Mathematics, Federal University of Technology, Owerri, Nigeria \\ *Corresponding author: scinyama2011@yahoo.com \\ Received Oct. 8, 2019
}

\begin{abstract}
AвSTRACT. In this study, we propose an SIR Mathematical model with vaccination and migration effect to analyze the behaviours of the transaction of Dengue when vaccination with dengvaxia has been carried out. There is no effective of Dengue disease. We introduce check and vaccination of immigrants as a measure in the model which can help to reduce the burden of Dengue disease. Basic reproduction number was calculated and used to determine whether the disease comes under control when $R_{0}<1$ and become more prevalent when $R_{0}>1$. Results in the work showed that disease-free equilibrium of the model is locally asymptotically stabe and the endemic equilibrium was proved to be globally asymptotically stable when $R_{0}<1$ using Lyapunov method. To understand more of the model we illustrate that with aid of simulation and from the first simulation it was revealed that the susceptibles mosqutoes remains in the population, when the susceptible mosquitoes bites any of the class which results in the spread of Dengue disease. The second simulation reveals that the population will be reduced over time, that is to say that for a period of 555 days the entire severely infected will go into extenction when $q_{1}=q_{2}=0$. Simulation also shows that with vaccination alone, the severely infected population can be reduced but can not be wiped out on the long run.
\end{abstract}

2010 Mathematics Subject Classification. 93A30.

Key words and phrases. dengue fever; transimission; disease-free equilibruim; vaccination; migrant.

\section{InTRODUCTION}

Whenever there is an incidence of an infectious disease in any population, people start to look for methods that are best to eradicate the disease or at least control the number of infected individual. Experiments for producing and testing those control measures, such as vaccination, quarantining the infected population, or use of antiviral drugs, are costly and time consuming, so any tool that will enable us to predict the outcome is highly valuable. Mathematical models are considered to be viable frameworks for describing the temporal dynamics and control of spread infectious diseases. Models in modern days play a crucial role in policy making, planning for emergency outbreaks risk assessment, evaluation of control programs and optimizing control methods, [2].

In the epidemics of Dengen, Delhi, recorded about more than 15000 cases of dengue outbreak in 2015. This was a worst of its kind since 2006 they recorded their first outbreak. Also in 2015, Hawaii, USA recorded a case of

DOI: 10.28924/APJM/7-3

(2020 Asia Pacific Journal of Mathematics 
outbreak with 181 infected individuals and transmission is still ongoing as of 2016. Other areas like; The Pacific island countries of Fiji, Tonga and French Polynesia have also recorded cases and are still recording.

Many mathematical models have been put in place to describe and analyze the behaviors of dengue disease. Most of these works which have appeared so far have successfully helped in understanding the dynamics of dengue fever (DF) and dengue hemorrhagic fever (DHF). Most of the works are channeled to different aspects of disease. For examples [8], [13], [16], [20] and [23] all developed an SIR epidemic model to show the mode of transmission of dengue disease considering both the human and mosquito population in their work.

$[6,7]$ developed an SEIR model to determine the influence of climatic condition such as rainfall on mode of transmission of dengue in Thailand. They showed that the spread of dengue in a particular country depends on the rate of rainfall in such a country. Another paper considered in this research was by [11] and a lot of insight has been obtained.

They determined and analyzed the stability of their model and performed simulations especially on the rainfall parameter. Their results were actually in agreement with analytic results obtained from their analysis.

Studies by [18] on the mode of transmission of the severe case of dengue using an SIR dynamic flow with age structure was carried out recently. Research shows that incubation periods in both the hosts and vectors have a great in?uence in the mode of transmission of dengue infection. So, different scholars like: [4], [17] and [22] with their mathematical models have studied dengue disease transmission dynamics with incubation periods in both human and vector.Their various works have shown the validity of this research discovery.

Most researchers believed that since dengue infection have no specific cure, that other measures can be put in place to reduce the burden of the transmission of the infection. Some of them include [9].

In 2017, [15] again studied a vector host epidemic and [14] believed that awareness campaign would help to reduce the prevalence of dengue infection. While some of them believed that prevention of the bites of mosquito (that is vector control measure) can as well reduce the rate of prevalence of the infection. So, to get rid of mosquitoes' bite, people can use mosquito repellents and nets. If infected hosts feel they have symptoms of the disease and approach the doctor in time for the supportive treatment, they can recover fast. This type of awareness can help controlling the disease. Again destroying the breeding sites of mosquitoes during their larval stage and killing them also helps to control dengue infection. Spray of insecticides may be applied to control larvae or adult mosquitoes which can transmit dengue viruses. [27] studied the demographic factors that in?uence equilibrium prevalence, and performed a sensitivity analysis on the basic reproduction number. Among all the measures used in this work, it was only two measures that showed great tendency of reducing the spread of the virus. These are introducing insecticide to the mosquito population and introducing vaccination on the human population. A stochastic model was also built to investigate the effects of some demographic factors. In the work of [9], they showed that awareness of the spread of dengue lowers the number of infectious individual, since the susceptible individual will take precautions against the bite of mosquito. However, only those who are reluctant about the information falls victim of the disease.

Also in 2016, [13] studied looked into the model of transmission with and without awareness campaign, where they made the assumption that in the case of awareness, that the humans population protects themselves against the infected mosquitor, (that is, they avoid the bite of mosquitoes by sleeping under net maybe). While on the 
other case of non-awareness there is a bite of human by the mosquitoes. The result of their work was not different from that of [9].

There are some studies which scholars have studied on model of dengue disease by applying control measures. The work was aimed at finding the effects of different control measures on the dengue disease development. They obtained the basic reproduction number $\mathrm{R}_{0}$ using Next Generation Operator method. Using this basic reproduction number, they studied the Stability of their model and performed sensitivity analysis. Their work shows that control on the vector drastically reduce the basic reproduction to be less than unity, thereby accounting for the stability of their work.

Again, since the introduction of Dengvaxia vaccine by [25], mathematical models have been used to study the impact of this vaccine in the mode of transmission of dengue.

In another work by [21], they studied the use of Dengvaxia to fight against the spread of dengue in Philippines and suggest that through appropriated pricing, that the rate of prevalence of dengue can be reduced and that there is possibility of having a stable price.

In another study, [19] suggested that mathematical models have to come in to support the already available data produced by epidemiology and clinics and that this can be possible in the case of studying the efficacy of the introduced vaccine. They also suggest that serotype of the virus; age structure, individuals, and severity of case are the factors that can affect the efficacy of vaccine. They therefore, see mathematical modelling as a valuable tool when it comes to policy-making and the issues of dengue vaccination cannot be exempted.

Study by [1] on the Impact of the dengue vaccine in the endemic countries taking into account four different age groups depending on their response to the introduced vaccine. Recently, [5] proposed an SIR mathematical model with which they looked at the mode of transmission of dengue fever when vaccination of the host is involved. In their work they considered only one strain of infection and assumed no age difference. They introduced a parameter to study the efficacy of the vaccination in their work. Of which their analysis result showed that the efficacy parameter appear in their basic reproduction number and it also contributes in the reduction of the basic reproduction number to be less than one (3.1).

\section{Model Formulation}

2.1. The assumptions of the Model. We made the following assumptions;

(1) A horizontal standard incidence with homogeneous mixing, this means that susceptible individuals only infected through biting of infected mosquitoes.

(2) The susceptible human population is increased by recruitment (birth and immigration) at a constant rate, $\Lambda_{H}$.

(3) That most individuals who are infected travels, also that there is no strict and constant screening exercise to reject infected individuals into the population under study, [26].

(4) Humans leave the population through natural death at constant rate $m u_{\mathrm{H}}$ and the severe infected humans $\left(\mathbf{I}_{H_{2}}\right)$ have an additional disease-induced death rate constant $\varepsilon$, [25].

(5) That recovered individuals acquires permanent immunity against re-infection (so that recovered individuals do not return to the susceptible human class). 
(6) The efficacy of the vaccine is not be $100 \%$, hence the vaccine is an imperfect one, [25].

(7) A fraction $\boldsymbol{\rho}$ of the recruited individuals are vaccinated while $(\mathbf{1}-\boldsymbol{\rho})$ are unvaccinated.

(8) The disease does not kill the infected mosquitoes while the susceptible ones leave the population through natural death, $\boldsymbol{\mu}_{\boldsymbol{m}}$.

(9) That the infected individuals recovers at a constant rate $\boldsymbol{\alpha}_{\boldsymbol{H}}$, however some fractions $\boldsymbol{k}$ due to complication fails to recover and become severely infected.

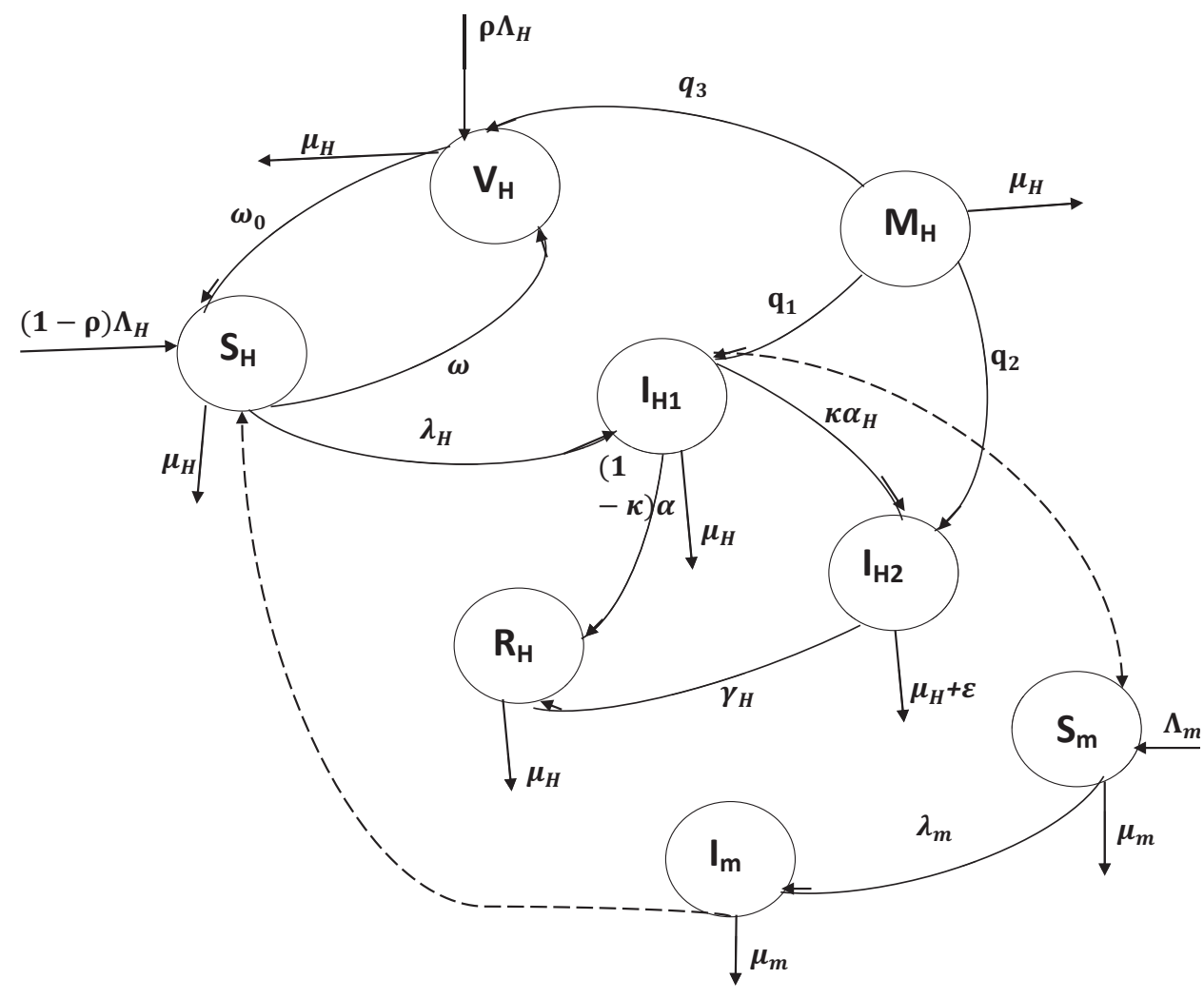

FIGURE 1. Schematic diagram of the model

where $\lambda_{H}=\frac{\beta_{H} I_{m}}{N_{H}}$ and $\lambda_{m}=\frac{\beta_{m}\left(I_{H 1}+\eta I_{H 2}\right)}{N_{H}}$ 


\subsection{Symbols and Parameters Description.}

\begin{tabular}{|l|l|}
\hline Symbols and Parameters & Description \\
\hline$V_{H}$ & Number of Vaccinated individual at time $\mathrm{t}$ \\
\hline$S_{H}$ & The number of susceptible human at time $\mathrm{t}$ \\
\hline$I_{H 1}$ & The number of common dengue individuals at time $\mathrm{t}$ \\
\hline$I_{H 2}$ & The number of severe infected individuals at time $\mathrm{t}$ \\
\hline$M$ & The number of immigrants at time $\mathrm{t}$ \\
\hline$R_{H}$ & The number of recovered human individual at time $\mathrm{t}$ \\
\hline$S_{m}$ & Number of susceptible mosquito at time $\mathrm{t}$ \\
\hline$I_{m}$ & Number of infected mosquito at time $\mathrm{t}$ \\
\hline$\Lambda_{H}$ & Recruitment rate of human \\
\hline$\Lambda_{m}$ & Recruitment rate of mosquito \\
\hline$\beta_{H}$ & Contact rate of human \\
\hline$\beta_{m}$ & Contact rate of mosquito \\
\hline$\alpha_{H}$ & The rate at which the infected individuals become severely infected \\
\hline$\kappa$ & Fraction of individual who become severely infected \\
\hline$\gamma_{H}$ & The rate at which severe infected individuals are recovering \\
\hline$\omega$ & The rate at which susceptible individuals are vaccinated \\
\hline$\omega_{0}$ & Vaccine weaning rate \\
\hline$q_{1}$ & The rate of flow from Migrated class to infected class \\
\hline$q_{2}$ & The rate of flow from Migrated class to severe infected class \\
\hline$q_{3}$ & The rate at which immigrants are vaccinated \\
\hline$\mu_{H}$ & Natural death rate of human \\
\hline$\mu_{m}$ & Natural death rate of mosquito \\
\hline$\rho$ & Fraction of recruited individual who are vaccinated \\
\hline$\varepsilon$ & Disease induced death rate \\
\hline & \\
\hline & \\
\hline
\end{tabular}




\section{Mathematical Model Formulation}

In this work, using the assumptions, symbols and parameter stated above we now formulate our Mathematical Model of dengue fever.

$$
\begin{aligned}
\frac{d V_{H}}{d t} & =\Lambda_{H} \rho+\omega S_{H}+q_{3} M-\left(\omega_{0}+\mu_{H}\right) V_{H} \\
\frac{d S_{H}}{d t} & =(1-\rho) \Lambda_{H}+\omega_{0} V_{H}-\frac{\beta_{H} I_{m} S_{H}}{N_{H}}-\left(\omega+\mu_{H}\right) S_{H} \\
\frac{d I_{H 1}}{d t} & =\frac{\beta_{H} I_{m} S_{H}}{N_{H}}+q_{1} M-\left(\alpha_{H}+\mu_{H}\right) I_{H 1} \\
\frac{d I_{H 2}}{d t} & =q_{2} M+k \alpha_{H} I_{H 1}-\left(\gamma_{H}+\mu_{H}+\varepsilon\right) I_{H 2} \\
\frac{d M}{d t} & =-\left(q_{1}+q_{2}+q_{3}+\mu_{H}\right) M \\
\frac{d R_{H}}{d t} & =\gamma_{H} I_{H 2}+(1-k) \alpha_{H} I_{H 1}-\mu_{H} R_{H} \\
\frac{d S_{m}}{d t} & =\Lambda_{m}-\frac{\beta_{m}\left(I_{H 1}+I_{H 2}\right) S_{m}}{N_{m}}-\mu_{m} S_{m} \\
\frac{d I_{m}}{d t} & =\frac{\beta_{m}\left(I_{H 1}+I_{H 2}\right) S_{m}}{N_{m}}-\mu_{m} I_{m}
\end{aligned}
$$

We consider in this work a basic deterministic dengue fever model which is sub-divided into two populations namely; $N_{H}=V_{H}+S_{H}+I_{H 1}+I_{H 2}+M+R_{H}$ and $N_{m}=S_{m}+I_{m}$ with the assumptions, the nature and time rate of transfer from one compartment to another as given above. We also consider the framework of the model to suit a dengue disease with permanent immunity on recovery from the infection. The model indicates that the passage of individuals is from Migrated class $M$, to the Vaccinated class, $V_{H}$, to the Susceptible human class, $S_{H}$, then to infective classes, $I_{H 1}$ to $I_{H 2}$ and ?nally to the recovery class, $R_{H}$. $S_{H}$ represents the number of individuals not yet infected with dengue virus at time $t$, or those susceptible to the disease.

While $V_{H}, I_{H 1}, I_{H 2}, M$ denote the number of vaccinated individuals, individuals infected with dengue fever at the primary stage, severely infectious individuals, Migrated individuals at time $t$, respectively, and $R_{H}$ represents the total population of recovered individuals at time $t . \Lambda_{H}$ is the recruitment rate into the human population, $\beta_{H}$ is the disease transmission coefficient for the human, $\mu_{H}$ is the natural death rate of the population, $\varepsilon$ is the death rate for disease of infectious individuals, $\rho$ represent the fraction of individual who are vaccinated at a time $t, \kappa$ is the fraction of individual who become severely infected while $1-\kappa$ denotes the fraction who recovered from the primary stage, $\omega_{0}$ denotes the vaccine weaning rate and $\gamma_{H}, \alpha_{H}, q_{1}, q_{2}, q_{3}$ and ware the state transition rates. We suppose that the recovered individuals have gained permanent immunity and can no longer be infected.

In this study, only female Aedes mosquitoes are considered as the transmission vector since only female mosquito bites human for blood meals. The female mosquitoes enter the susceptible class through breeding, at a rate $\Lambda_{\mathrm{M}}$. We assume the disease does not kill the infected mosquitoes while the susceptible ones leave the population through natural death, $\mu_{\mathrm{m}}$. A susceptible mosquito can only be infected by biting an already infected human.

\section{Analysis of the Model}

\subsection{Invariant Region}


The total population $\mathbf{N}_{\boldsymbol{H}}$ and $\mathbf{N}_{\boldsymbol{m}}$ of both human and mosquito, can be determined by the differential equations

$$
\left.\begin{array}{l}
\frac{d N_{H}}{d t}=\frac{d V_{H}}{d t}+\frac{d S_{H}}{d t}+\frac{d I_{H 1}}{d t}+\frac{d I_{H 2}}{d t}=\frac{d M}{d t}=\frac{d R_{H}}{d t} \\
\frac{d N m}{d t}=\frac{S_{m}}{d t}+\frac{d I_{m}}{d t}
\end{array}\right\}
$$

Summing the equations of the system (3.1) rigorously, we obtain $\frac{d N_{H}}{d t}=\Lambda_{H}-\mu_{H}\left(V_{H}+S_{H}+I_{H 1}+I_{H 2}+M+\right.$ $\left.R_{H}\right)-\varepsilon I_{H 2}$

$$
\begin{gathered}
\frac{d N_{H}}{d t}=\Lambda_{H}-\mu_{H} N_{H}-\varepsilon I_{H 2} \leq \Lambda_{H}-\mu_{H} N_{H} \\
\Rightarrow \frac{d N_{H}}{d t} \leq \Lambda_{H}-\mu_{H} N_{H},
\end{gathered}
$$

Re-writing we have

$$
\frac{d N_{H}}{d t}=\mu_{H} N_{H} \leq \Lambda_{H}
$$

Solving the differential inequality (3.2),

$$
\frac{d}{d t}\left(N_{H} e^{\mu_{H} t}\right) \leq \Lambda_{H} e^{\mu_{H} t}
$$

Integrating we have

$$
N_{H} e^{\mu_{H} t} \leq \int \Lambda_{H} e^{\mu_{H} t} d t
$$

This implies $N_{H} e^{\mu_{H} t} \leq \frac{\Lambda_{H}}{\mu_{H}} e^{\mu_{H} t}+C$

Hence, $N_{H} \leq \frac{\Lambda_{H}}{\mu_{H}}+C e^{-\mu_{H} t}$

$$
\text { As } t \rightarrow \infty, \quad N_{H} \leq \frac{\Lambda_{H}}{\mu_{H}}
$$

This implies that, the total human population $N_{H}$ varies with timet. And in an entirely disease free population, the total human population size $N_{H}$ converges to $\frac{\Lambda_{H}}{\mu_{H}}$.

We can as well in a similar way, show that the total mosquito population size $N_{m}$ varies with timet and then, in an entirely disease free population, the total mosquito population size $N_{m}$ will converge to $\frac{\Lambda_{m}}{\mu_{m}}$. Hence, we are studying our model (3.1) in the feasible set given by

$$
\Omega=\left\{\left(V_{H}, S_{H}, I_{H 1}, I_{H 2}, M, R_{H}, S_{m}, I_{m}\right) \in \mathrm{R}_{+}^{8}: V_{H}, S_{H}, I_{H 1}, I_{H 2}, M, R_{H}, S_{m}, I_{m} \geq 0 ; N_{H} \leq \frac{\Lambda_{H}}{\mu_{H}} ; N_{m} \leq \frac{\Lambda_{m}}{\mu_{m}}\right\},
$$

which is a positively invariant. Therefore our model is epidemiologically meaningful and well-posed in the domainO. Again, in this domain it is important to study the dynamics of model (3.1), since there exist a unique and continuation solution of the system. Also, it is very veritable to show that the solution set $\left\{V_{H}, S_{H}, I_{H 1}, I_{H 2}, M, R_{H}, S_{m}, I_{m}\right\}(t)$ of our model (3.1) are all positive, for all $\left(\left(V_{H}, S_{H}, I_{H 1}, I_{H 2}, M, R_{H}, S_{m}, I_{m}\right) \geq\right.$ $0) \in \Omega, t>0$. This will be seen in the next section that follows.

\subsection{Positivity of Solutions}

We show that all the variables in the model equation are non-negative.

\section{Theorem 4.1(Positivity of Solutions)}

We set, the initial data for the model (3.1) be $V_{H}(0)>0, S_{H}(0)>0, I_{H 1}(0)>0, I_{H 2}(0)>0, M(0)>0, R_{H}(0)>$ $0, S_{m}(0)>0, I_{m}>0$.

Then, the solution set $\left\{V_{H}(t), S_{H}(t), I_{H 1}(t), I_{H 2}(t), M(t), R_{H}(t), S_{m}(t), I_{m}(t)\right\}$ of the system (3.1) with positive initial data, remains positive when $t>0$. 


\section{Proof:}

From the first equation of model (3.1);

$$
\frac{d V_{H}(t)}{d t}=\Lambda_{H} \rho+\omega S_{H}+q_{3} M-\left(\omega_{0}+\mu_{H}\right) V_{H} \geq-\left(\omega_{0}+\mu_{H}\right) V_{H}
$$

Then $\frac{d V_{H}(t)}{d t} \geq-\left(\omega_{0}+\mu_{H}\right) V_{H}$

$$
\Rightarrow \frac{d V_{H}(t)}{V_{H}} \geq-\left(\omega_{0}+\mu_{H}\right) d t
$$

Integrating, we have,

$$
\begin{gathered}
\operatorname{In} V_{H} \geq-\left(\omega_{0}+\mu_{H}\right) t+K \\
V_{H}(t) \geq K e^{-\left(\omega_{0}+\mu_{H}\right) t}
\end{gathered}
$$

At $t=0$ we have that $V_{H}(0) \geq K$

Hence $V_{H}(t) \geq V_{H}(0) e^{-\left(\omega_{0}+\mu_{H}\right) t} \geq 0$

Since $\left(\omega_{0}+\mu_{H}\right)>0$

For the second equation

$$
\frac{d S_{H}(t)}{d t}=\Lambda_{H}(1-\rho)+\omega_{0} V_{H}-\frac{\beta_{H} I_{m} S_{H}}{N_{H}}-\left(\omega+\mu_{H}\right) S_{H} \geq-\left(\omega+\mu_{H}\right) S_{H}
$$

Then $\frac{d S_{H}(t)}{d t} \geq-\left(\omega+\mu_{H}\right) S_{H}$

This implies that; $\frac{d S_{H}(t)}{S_{H}} \geq-\left(\omega+\mu_{H}\right) d t$

Integrating, we have,

$$
\begin{aligned}
& \operatorname{InS}_{H}(t) \geq-\left(\omega+\mu_{H}\right) t+K \\
& S_{H}(t) \geq K e^{-\left(\omega+\mu_{H}\right) t}
\end{aligned}
$$

At $t=0$ we have that $S_{H}(0) \geq K$

Hence $S_{H}(t) \geq S_{H}(0) e^{-\left(\omega+\mu_{H}\right) t} \geq 0$

Since $\left(\omega+\mu_{H}\right)>0$

For the third equation;

$$
\frac{d I_{H 1}(t)}{d t}=\frac{\beta_{H} I_{m} S_{H}}{N_{H}}+q_{1} M-\left(\alpha_{H}+\mu_{H}\right) I_{H 1} \geq-\left(\alpha_{H}+\mu_{H}\right) I_{H 1}
$$

Then $\frac{d I_{H 1}(t)}{d t} \geq-\left(\alpha_{H}+\mu_{H}\right) I_{H 1}$

$$
\frac{d I_{H 1}(t)}{I_{H 1}} \geq-\left(\alpha_{H}+\mu_{H}\right) d t
$$


Integrating, we have,

$$
\begin{aligned}
& \operatorname{InI}_{H 1} \geq-\left(\alpha_{H}+\mu_{H}\right) t+K \\
& I_{H 1}(t) \geq K e^{-\left(\alpha_{H}+\mu_{H}\right) t}
\end{aligned}
$$

At $t=0$ we have that $I_{H 1}(0) \geq K$

Hence $I_{H 1}(t) \geq I_{H 1}(0) e^{-\left(\alpha_{H}+\mu_{H}\right) t} \geq 0$

Since $\left(\alpha_{H}+\mu_{H}\right)>0$

For the fourth equation;

$$
\frac{d I_{H 2}(t)}{d t}=q_{2} M+\kappa \alpha_{H} I_{H 1}-\left(\gamma_{H}+\mu_{H}+\varepsilon\right) I_{H 2} \geq-\left(\gamma_{H}+\mu_{H}+\varepsilon\right) I_{H 2}
$$

Then $\frac{d I_{H 2}(t)}{d t} \geq-\left(\gamma_{H}+\mu_{H}+\varepsilon\right) I_{H 2}$

That implies; $\frac{d I_{H 2}(t)}{I_{H 2}} \geq-\left(\gamma_{H}+\mu_{H}+\varepsilon\right) d t$

Integrating we have

$$
\begin{aligned}
& \operatorname{InI}_{H_{2}} \geq-\left(\gamma_{H}+\mu_{H}+\varepsilon\right) t+K \\
& I_{H 2}(t) \geq K e^{-\left(\gamma_{H}+\mu_{H}+\varepsilon\right) t}
\end{aligned}
$$

At $t=0$ we have that $I_{H 2}(0) \geq K$

Hence $I_{H 2}(t) \geq I_{H 2}(0) e^{-\left(\gamma_{H}+\mu_{H}+\varepsilon\right) t} \geq 0$

Since $\left(\gamma_{H}+\mu_{H}+\varepsilon\right)>0$

For the fifth equation of system (3.1)

$$
\begin{gathered}
\frac{d M(t)}{d t}=-\left(q_{1}+q_{2}+q_{3}+\mu_{H}\right) M \\
\frac{d M(t)}{M}=-\left(q_{1}+q_{2}+q_{3}+\mu_{H}\right) d t
\end{gathered}
$$

Integrating, we have,

$$
\begin{aligned}
& \text { InM }=-\left(q_{1}+q_{2}+q_{3}+\mu_{H}\right) t+K \\
& M(t)=K e^{-\left(q_{1}+q_{2}+q_{3}+\mu_{H}\right) t}
\end{aligned}
$$

At $t=0$ we have that $M(0)=K$

Hence $M(t)=M(0) e^{-\left(q_{1}+q_{2}+q_{3}+\mu_{H}\right) t} \geq 0$

Since $\left(q_{1}+q_{2}+q_{3}+\mu_{H}\right)>0$

For the sixth equation

$$
\frac{d R_{H}(t)}{d t}=\gamma_{H} I_{H 2}+(1-\kappa) \alpha_{H} I_{H 1}-\mu_{H} R_{H} \geq-\mu_{H} R_{H}
$$

Then $\frac{d R_{H}(t)}{d t} \geq-\mu_{H} R_{H}$

$$
\frac{d R_{H}(t)}{R_{H}} \geq-\mu_{H} d t
$$


Integrating, we have,

$$
\begin{aligned}
& \operatorname{In}_{H} \geq-\mu_{H} t+K \\
& R_{H} \geq K e^{-\mu_{H} t}
\end{aligned}
$$

At $t=0$ we have that $R_{H}(0) \geq K$

Hence $R_{H}(t) \geq R_{H}(0) e^{-\mu_{H} t}>0$

Since $\mu_{H}>0$

For the seventh equation

$$
\frac{d S_{m}(t)}{d t}=\Lambda_{m}-\frac{\beta_{m}\left(I_{H 1}+I_{H 2}\right) S_{m}}{N_{H}}-\mu_{m} S_{m} \geq-\mu_{m} S_{m}
$$

Then $\frac{d S_{m}(t)}{d t} \geq-\mu_{m} S_{m}$

$$
\frac{d S_{m}(t)}{S_{m}} \geq-\mu_{m} d t
$$

Integrating, we have,

$$
\begin{aligned}
& \text { In } S_{m} \geq-\mu_{m} t+K \\
& S_{m} \geq K e^{-\mu_{m} t}
\end{aligned}
$$

At $t=0$ we have that $S_{m}(0) \geq K$

Hence $S_{m}(t) \geq S_{m}(0) e^{-\mu_{m} t}>0$

Since $\mu_{m}>0$

For the eighth equation

$$
\frac{d I_{m}(t)}{d t}=\frac{\beta_{m}\left(I_{H 1}+I_{H 2}\right) S_{m}}{N_{H}}-\mu_{m} I_{m} \geq-\mu_{m} I_{m}
$$

Then $\frac{d I_{m}(t)}{d t} \geq-\mu_{m} I_{m}$

$$
\frac{d I_{m}(t)}{I_{m}} \geq-\mu_{m} d t
$$

Integrating, we have,

$$
\begin{aligned}
& \text { In } I_{m} \geq-\mu_{m} t+K \\
& I_{m} \geq K e^{-\mu_{m} t}
\end{aligned}
$$

At $t=0$ we have that $I_{m}(0) \geq K$

Hence $I_{m}(t) \geq I_{m}(0) e^{-\mu_{m} t}>0$

Since $\mu_{m}>0$

Hence for all $t>0$ the solution set $\left\{V_{H}(t), S_{H}(t), I_{H 1}(t), I_{H 2}(t), M(t), R_{H}(t), S_{m}(t), I_{m}(t)\right\}$ are all non-negative. 
In this section, we analysed system (3.1) in order to obtain the equilibrium points of the system and its stability. Let $E=\left(V_{H}^{*}, S_{H}^{*}, I_{H 1}^{*}, I_{H 2}^{*}, M^{*}, R_{H}^{*}, S_{m}^{*}, I_{m}^{*}\right)$ be the steady-state of system (1). At the steady states, weset each of the differential equations of the model (3.1) equal to zero. Hence at the equilibrium point;

$$
\left(\frac{d V_{H}}{d t}=\frac{d S_{H}}{d t}=\frac{d I_{H 1}}{d t}=\frac{d I_{H 2}}{d t}=\frac{d M}{d t}=\frac{d R_{H}}{d t}=\frac{d S_{m}}{d t}=\frac{d I_{m}}{d t}\right)=0
$$

This means;

$$
\begin{aligned}
& \Lambda_{H} \rho+\omega S_{H}+q_{3} M-\left(\omega_{0}+\mu_{H}\right) V_{H}=0 \\
& (1-\rho) \Lambda_{H}+\omega_{0} V_{H}-\frac{\beta_{H} I_{m} S_{H}}{N_{H}}-\left(\omega+\mu_{H}\right) S_{H}=0 \\
& \frac{\beta_{H} I_{m} S_{H}}{N_{H}}+q_{1} M-\left(\alpha_{H}+\mu_{H}\right) I_{H 1}=0 \\
& q_{2} M+k \alpha_{H} I_{H 1}-\left(\gamma_{H}+\mu_{H}+\varepsilon\right) I_{H 2}=0 \\
& -\left(q_{1}+q_{2}+q_{3}+\mu_{H}\right) M=0 \\
& \gamma_{H} I_{H 2}+(1-k) \alpha_{H} I_{H 1}-\mu_{H} R_{H}=0 \\
& \Lambda_{m}-\frac{\beta_{m}\left(I_{H 1}+I_{H 2}\right) S_{m}}{N_{m}}-\mu_{m} S_{m}=0 \\
& \frac{\beta_{m}\left(I_{H 1}+I_{H 2}\right) S_{m}}{N_{m}}-\mu_{m} I_{m}=0
\end{aligned}
$$

Solving system (3.3), obviously $\left(q_{1}+q_{2}+q_{3}+\mu_{H}\right) \neq 0$ that means $M=0$

$$
\left.\begin{array}{rl}
V_{H}^{*} & =\frac{\Lambda_{H} \rho+\omega S_{H}}{\omega_{0}+\mu_{H}} \\
S_{H}^{*} & =\frac{(1-\rho) \Lambda_{H}+\omega_{0} V_{H}}{\frac{\beta_{H} I_{m}}{N_{H}}+\omega+\mu_{H}} \\
I_{H 1}^{*} & =\frac{\beta_{H} I_{m}^{*} S_{H}^{*}}{\alpha_{H}+\mu_{H}} \\
I_{H 2}^{*} & =\frac{k \alpha_{H} I_{H 1}^{*}}{\gamma_{H}+\mu_{H}+\varepsilon} \\
M^{*} & =0 \\
R_{H}^{*} & =\frac{\gamma_{H} I_{H 2}^{*}+(1-k) \alpha_{H} I_{H 1}^{*}}{\mu_{H}} \\
S_{m}^{*} & =\frac{\Lambda_{m}}{\mu_{m}+\frac{\beta_{m}\left(I_{H 1}^{*}+I_{H 2}^{*}\right)}{N_{H}}} \\
I_{M}^{*} & =\frac{\beta_{m}\left(I_{H 1}^{*}+I_{H 2}^{*}\right) S_{m}}{\mu_{m} N_{H}}
\end{array}\right\}
$$

The population will never go into extinction, provided that there is constant human recruitment at the rate of $\boldsymbol{\Lambda}_{\boldsymbol{H}}$ and also constant breeding of mosquito at the rate of $\Lambda_{m}$. This means that, there is no trivial solution in our system, thus, $\left(V_{H}^{*}, S_{H}^{*}, I_{H 1}^{*}, I_{H 2}^{*}, M^{*}, R_{H}^{*}, S_{m}^{*}, I_{m}^{*}\right) \neq(0,0,0,0,0,0,0,0)$.

\subsubsection{Disease Free Equilibrium (DFE) of the Model}

The model system (3.1) has a steady state in the absence of dengue fever, that is, $I_{H 1}^{0}=I_{H 2}^{0}=M^{0}=I_{m}^{0}=0$. Hence, the disease-free equilibrium (DFE) denoted as $E^{0}$, of the model system (3.1) is gotten by Substituting $I_{H 1}^{0}=I_{H 2}^{0}=M^{0}=I_{m}^{0}=0$ into (3.3).

Hence the disease free equilibrium of system (3.1) of equation is given by

$$
E^{0}=\left(V_{H}^{0}, S_{H}^{0}, I_{H 1}^{0}, I_{H 2}^{0}, M^{0}, R_{H}^{0}, S_{m}^{0}, I_{m}^{0}\right)=\left(\frac{\Lambda_{H}\left(\omega+\mu_{H} \rho\right)}{\mu_{H}\left(\omega_{0}+\omega+\mu_{H}\right)}, \frac{\Lambda_{H}\left[\omega_{0}+\mu_{H}(1-\rho)\right]}{\mu_{H}\left(\omega_{0}+\omega+\mu_{H}\right)}, 0,0,0,0, \frac{\Lambda_{m}}{\mu_{m}}, 0\right)
$$

\subsubsection{The Model Basic Reproduction Number}

The local stability is established by using the next generation operator method on the system.

The basic reproduction number $R_{0}$ is defined as the effective number of secondary infections caused by an infected individual during his/her entire period of infectiousness, [24]. 
When $R_{0}<0$, it implies that each individual produces, on average, less than one new infected individual and hence the disease dies out with time. On the other hand, when $R_{0}>0$, it means each individual produces more than one new infected individual and hence the disease is able to invade the susceptible population. However, $R_{0}=0$ is the threshold below which the generation of secondary cases is insufficient to maintain the infection with human community. The basic reproduction number cannot be determined from the structure of the mathematical model alone, but depends on the de?nition of infected and uninfected compartments.

To obtainthis basic reproduction number $\mathrm{R}_{0}$, we take the dominant eigenvalue or spectral radius of $\rho\left(F_{i} V^{-1}\right)$ where $F_{i}=\left[\frac{\partial f_{i}\left(E^{0}\right)}{\partial X_{j}}\right]$ and $V_{i}=\left[\frac{\partial v_{i}\left(E^{0}\right)}{\partial X_{j}}\right]$

$f_{i}$ is the rate at which new infection appears in each of the diseaseclasses " $i$ " and $v_{i}$ is the rate at which individuals are transferred in and out of the disease classes and it is given by " $v_{i}=v_{i}{ }^{-}-v_{i}{ }^{+\prime}$

$v_{i}{ }^{-}$is the rate at which individuals are transferred out of the diseaseclass $i$,

$v_{i}{ }^{+}$is the rate at which individuals are transferredinto the diseaseclass $i$, by any other means,

$E^{0}$ is the disease free equilibrium.

Then by linearizing $f_{i}$ and $v_{i}$, and evaluating at the disease free equilibrium point we obtain the associated matrices $F_{i}$ and $V_{i}$ respectively.

The disease classesof model (3.1) are $I_{H 1}, I_{H 2}, M$ and $I_{m}$ hence a straightforward calculation gives $f(x)=\left(\begin{array}{c}\frac{\beta_{H} S_{H} I_{m}}{N_{H}} \\ 0 \\ 0 \\ \frac{\beta_{m} S_{m}\left(I_{H 1}+I_{H 2}\right)}{N_{H}}\end{array}\right)$ and $v(x)=\left(\begin{array}{l}\left(\mu_{H}+\alpha_{H}\right) I_{H 1}-q_{1} M \\ \left(\gamma_{H}+\mu_{H}+\varepsilon\right) I_{H 2}-\kappa \alpha_{H} I_{H 1}-q_{2} M \\ \left(q_{1}+q_{2}+q_{3}+\mu_{H}\right) M \\ \mu_{m} I_{m} \\ \end{array}\right)$

Then $F$ and $V$ which is the Jacobian of $f$ and $v$ evaluated at the DFE $E^{0}$ becomes

$$
F\left(E^{0}\right)=\left(\begin{array}{cccc}
0 & 0 & 0 & \frac{\beta_{H}\left[\omega_{0}+\mu_{H}(1-\rho)\right]}{\omega_{0}+\omega+\mu_{H}} \\
0 & 0 & 0 & 0 \\
0 & 0 & 0 & 0 \\
\frac{\beta_{m} \Lambda_{m} \mu_{H}}{\Lambda_{H} \mu_{m}} & \frac{\beta_{m} \Lambda_{m} \mu_{H}}{\Lambda_{H} \mu_{m}} & 0 & 0
\end{array}\right)
$$




$$
V\left(E^{0}\right)=\left(\begin{array}{cccc}
\alpha_{H}+\mu_{H} & 0 & -q_{1} & 0 \\
-\kappa \alpha_{H} & \gamma_{H}+\mu_{H}+\varepsilon & -q_{2} & 0 \\
0 & 0 & q_{1}+q_{2}+q_{3}+\mu_{H} & 0 \\
0 & 0 & 0 & \mu_{m}
\end{array}\right)
$$

After rigorous computation we obtain;

$$
V^{-1}=\left(\begin{array}{cccc}
\frac{1}{\alpha_{H}+\mu_{H}} & 0 & \frac{q_{1}}{\left(q_{1}+q_{2}+q_{3}+\mu_{H}\right)\left(\alpha_{H}+\mu_{H}\right)} & 0 \\
\frac{k \alpha_{H}}{\left(\alpha_{H}+\mu_{H}\right)\left(\varepsilon+\gamma_{H}+\mu_{H}\right)} & \frac{1}{\left(\varepsilon+\gamma_{H}+\mu_{H}\right)} & \frac{q_{2} \alpha_{H} \mu_{m}+q_{1} k \alpha_{H} \mu_{m}+q_{2} \mu_{H} \mu_{m}}{\left(q_{1}+q_{2}+q_{3}+\mu_{H}\right)\left(\alpha_{H}+\mu_{H}\right)\left(\varepsilon+\gamma_{H}+\mu_{H}\right) \mu_{m}} & 0 \\
0 & 0 & \frac{1}{\left(q_{1}+q_{2}+q_{3}+\mu_{H}\right)} & 0 \\
0 & 0 & 0 & \frac{1}{\mu_{m}}
\end{array}\right) \text { The basic reproduction }
$$

number is given by $R_{0}=\rho\left(F V^{-1}\right)$, that is, the highest eigenvalue of $F V^{-1}$ is

$$
\begin{gathered}
R_{0}=\sqrt{\frac{\beta_{H} \beta_{m} \Lambda_{m} \mu_{H}\left[\omega_{0}+\mu_{H}(1-\rho)\right]\left(\mu_{H}+\kappa \alpha_{H}+\gamma_{H}+\varepsilon\right)}{\mu_{m}^{2} \Lambda_{H}\left(\alpha_{H}+\mu_{H}\right)\left(\mu_{H}+\gamma_{H}+\varepsilon\right)\left(\omega_{0}+\omega+\mu_{H}\right)}} \\
R_{0}^{2}=\frac{\beta_{H} \beta_{m} \Lambda_{m} \mu_{H}\left[\omega_{0}+\mu_{H}(1-\rho)\right]\left(\mu_{H}+\kappa \alpha_{H}+\gamma_{H}+\varepsilon\right)}{\mu_{m}^{2} \Lambda_{H}\left(\alpha_{H}+\mu_{H}\right)\left(\mu_{H}+\gamma_{H}+\varepsilon\right)\left(\omega_{0}+\omega+\mu_{H}\right)}
\end{gathered}
$$

Hence it follows that $R_{0}={\sqrt{R_{H} \times R_{m}}}_{m}$ where

$R_{H}=\frac{\beta_{H} \mu_{H}\left[\omega_{0}+\mu_{H}(1-\rho)\right]\left(\mu_{H}+\kappa \alpha_{H}+\gamma_{H}+\varepsilon\right)}{\Lambda_{H}\left(\alpha_{H}+\mu_{H}\right)\left(\mu_{H}+\gamma_{H}+\varepsilon\right)\left(\omega_{0}+\omega+\mu_{H}\right)}$ and $R_{m}=\frac{\beta_{m} \Lambda_{m}}{\mu_{m}^{2}}$

where $R_{H}$ tells about the number of humans that one infectious mosquito infects during its period of infectiousness in an entirely susceptible human population and $R_{m}$ gives us the information about the average number of mosquitoes that one infectious individual can infect during his/her period of infections in an entirely susceptible mosquito population.

\subsubsection{Stability of the Disease Free Equilibrium $\left(E^{0}\right)$}

Theorem 3.1: The disease free equilibrium, $E^{0}$ is locally asymptotically stable if $R_{0}<1$ and unstable if $R_{0}>1$.

Proof;

We prove the locally asymptotically stability of the disease free equilibrium $E^{0}$ of model (3.1) using linearization approach.

First we considered the subsystem of model (3.1)

$$
\begin{aligned}
& \frac{d V_{H}}{d t}=\Lambda_{H} \rho+\omega S_{H}+q_{3} M-\left(\omega_{0}+\mu_{H}\right) V_{H} \\
& \frac{d S_{H}}{d t}=(1-\rho) \Lambda_{H}+\omega_{0} V_{H}-\frac{\beta_{H} I_{m} S_{H}}{N_{H}}-\left(\omega+\mu_{H}\right) S_{H} \\
& \frac{d I_{H 1}}{d t}=\frac{\beta_{H} I_{m} S_{H}}{N_{H}}+q_{1} M-\left(\alpha_{H}+\mu_{H}\right) I_{H 1} \\
& \frac{d I_{H 2}}{d t}=q_{2} M+\kappa \alpha_{H} I_{H 1}-\left(\gamma_{H}+\mu_{H}+\varepsilon\right) I_{H 2} \\
& \frac{d R_{H}}{d t}=\gamma_{H} I_{H 2}+(1-\kappa) \alpha_{H} I_{H 1}-\mu_{H} R_{H} \\
& \frac{d M}{d t}=-\left(q_{1}+q_{2}+q_{3}+\mu_{H}\right) M
\end{aligned}
$$

With Jacobian; 


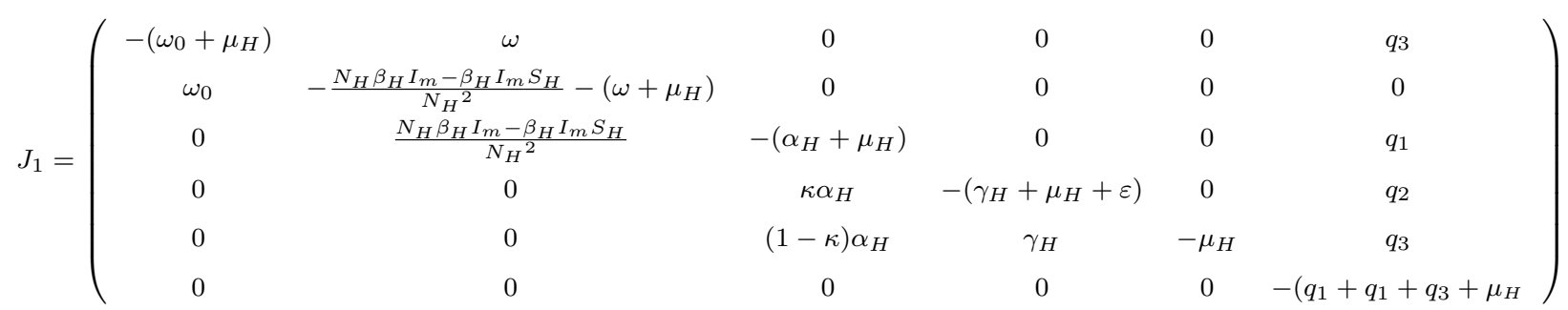

At the disease free equilibrium $J_{1}$ becomes,

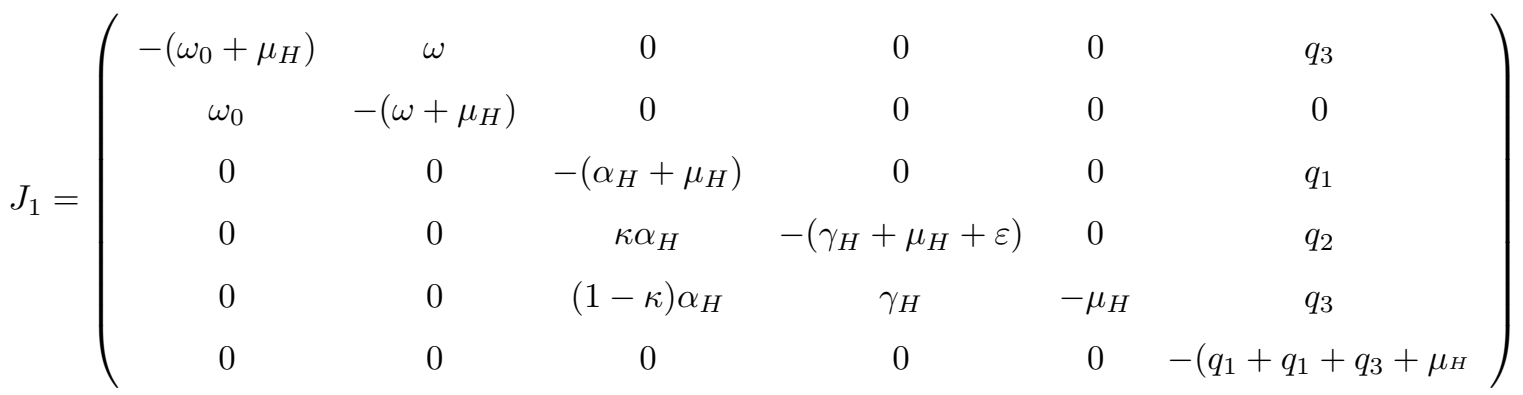

Hence the characteristics equation of the above Jacobian is $\left|\lambda I-J_{1}\right|=0$ where $I$ is $6 \times 6$ identity

$$
\left|J_{1}-I \lambda\right|=\left|\begin{array}{cccccc}
-\left(\omega_{0}+\mu_{H}\right)-\lambda & -\omega & 0 & 0 & 0 & -q_{3} \\
-\omega_{0} & -\left(\omega+\mu_{H}\right)-\lambda & 0 & 0 & 0 & 0 \\
0 & 0 & -\left(\alpha_{H}+\mu_{H}\right)-\lambda & 0 & 0 & -q_{1} \\
0 & 0 & -k \alpha_{H} & -\left(\gamma_{H}+\mu_{H}+\varepsilon\right)-\lambda & 0 & -q_{2} \\
0 & 0 & (1-k) \alpha_{H} & -\gamma_{H} & -\mu_{H}-\lambda & -q_{3} \\
0 & 0 & 0 & 0 & 0 & -\left(q_{1}+q_{2}+q_{3}+\mu_{H}\right)
\end{array}\right|
$$

After

rigorous computation we obtain;

$\left[\lambda+\left(\alpha_{H}+\mu_{H}\right)\right]\left[\lambda+\left(q_{1}+q_{1}+q_{3}+\mu_{H}\right)\right]\left[\lambda+\left(\gamma_{H}+\mu_{H}+\varepsilon\right)\right]\left[\lambda+\mu_{H}\right]\left[\lambda^{2}+\left(\omega+\omega_{0}+2 \mu_{H}\right) \lambda+\mu_{H}\left(\omega+\omega_{0}+\mu_{H}\right)\right]=0$

It is obvious that $\lambda_{1}=-\left(\alpha_{H}+\mu_{H}\right), \lambda_{2}=-\left(q_{1}+q_{1}+q_{3}+\mu_{H}\right), \lambda_{3}=-\left(\gamma_{H}+\mu_{H}+\varepsilon\right), \lambda_{4}=-\mu_{H}$ and

$$
\lambda^{2}+\left(\omega+\omega_{0}+2 \mu_{H}\right) \lambda+\mu_{H}\left(\omega+\omega_{0}+\mu_{H}\right)=0
$$

Letting

$$
\begin{gathered}
A_{2}=1 \\
A_{1}=\omega+\omega_{0}+2 \mu_{H} \\
A_{0}=\mu_{H}\left(\omega+\omega_{0}+2 \mu_{H}\right) \\
A_{2} \lambda^{2}+A_{1} \lambda+A_{0}=0
\end{gathered}
$$

We now employ the Routh-Hurwitz stability criterion according to [12] which states that all the roots of the characteristics equation (3.4) have negative real parts if and only the co-efficient $A_{i}$ are positive and matrices $H_{i}>0$ for $i=0,1,2$.

Hence from (3.4) obviously,

$A_{2}>0, A_{1}>0$ and $A_{0}>0$

Again, the Hurwitz matrice $H=\left(\begin{array}{cc}A_{1} & 0 \\ A_{0} & A_{2}\end{array}\right)$ 
With $H_{1}=\left|A_{1}\right|=A_{1}>0, H_{2}=\left|\begin{array}{cc}A_{1} & 0 \\ A_{0} & A_{2}\end{array}\right|=A_{1} A_{2}>0$

Also if we consider the subsystem of model (3.1)

$$
\begin{aligned}
\frac{d S_{m}}{d t} & =\Lambda_{m}-\frac{\beta_{m}\left(I_{H 1}+I_{H 2}\right) S_{m}}{N_{H}}-\mu_{m} S_{m} \\
\frac{d I_{m}}{d t} & =\frac{\beta_{m}\left(I_{H 1}+I_{H 2}\right) S_{m}}{N_{H}}-\mu_{m} I_{m}
\end{aligned}
$$

With Jacobian

$$
J_{2}=\left(\begin{array}{cc}
-\frac{\beta_{m}\left(I_{H 1}+I_{H 2}\right)}{N_{H}}-\mu_{m} & 0 \\
\frac{\beta_{m}\left(I_{H 1}+I_{H 2}\right)}{N_{H}} & -\mu_{m}
\end{array}\right)
$$

At the disease free equilibrium we, have $J_{2}=\left(\begin{array}{cc}-\mu_{m} & 0 \\ 0 & -\mu_{m}\end{array}\right)$

The characteristics equation $\left|\lambda I-J_{2}\right|=0$ where $I$ is a $2 \times 2$ identity matrix, becomes;

$$
\left(\lambda+\mu_{m}\right)\left(\lambda+\mu_{m}\right)=0
$$

Hence we have that $\lambda_{1}=\lambda_{2}=-\mu_{m}$

Therefore, this show that all the eigenvalues of the Jacobian matrices $J_{1}$ and $J_{2}$ have negative real parts, hence the disease free equilibrium is locally asymptotically stable.

\subsubsection{Global Asymptotic Stability for Disease-Free Equilibrium}

Theorem 3.3.4: If $R_{0} \leq 1$ for the model (3.1), then the infection-free equilibrium state $E^{0}$ is the only non-negative equilibrium state of this model, and it is globally asymptotically stable.

\section{Proof;}

Define the Lyapunov function;

$$
L=C_{1} I_{H 1}+C_{2} I_{H 2}+C_{3} M+C_{4} I_{m}
$$

where $\frac{m_{1}\left(\mu_{H}+\gamma_{H}+\kappa \alpha_{H}+\varepsilon\right)}{R_{0}\left(\alpha_{H}+\mu_{H}\right)\left(\mu_{H}+\gamma_{H}+\varepsilon\right)}=C_{1}$

$$
\begin{gathered}
\frac{m_{1}}{R_{0}\left(\mu_{H}+\gamma_{H}+\varepsilon\right)}=C_{2} \\
\frac{m_{1}\left[q_{2}\left(\alpha_{H}+\mu_{H}\right)+q_{1}\left(\mu_{H}+\gamma_{H}+\kappa \alpha_{H}+\varepsilon\right)\right]}{R_{0}\left(q_{1}+q_{2}+q_{3}+\mu_{H}\right)\left(\alpha_{H}+\mu_{H}\right)\left(\mu_{H}+\gamma_{H}+\varepsilon\right)}=C_{3}
\end{gathered}
$$

$\frac{1}{\mu_{m}}=C_{4}$, and $m_{1}=\frac{\beta_{m} \Lambda_{m} \mu_{H}}{\Lambda_{H} \mu_{m}^{2}}$

Differentiating (3.5) with respect to' $t^{\prime}$ we obtain;

$$
\frac{d L}{d t}=C_{1} \frac{d I_{H 1}}{d t}+C_{2} \frac{d I_{H 2}}{d t}+C_{3} \frac{d M}{d t}+C_{4} \frac{d I_{m}}{d t}
$$

Substituting the values of $c_{1}, c_{2}, c_{3}, c_{4}$ and system (3.1) into (3.6), we have collecting like terms and re-arranging (3.6)

$$
\frac{d L}{d t}=\left(\frac{\left.m_{1} \beta_{H} S_{H}\left(\mu_{H}+\gamma_{H}+k \alpha_{H}+\varepsilon\right)\right]}{N_{H} R_{0}\left(\alpha_{H}+\mu_{H}\right)\left(\gamma_{H}+\mu_{H}+\varepsilon\right)}-1\right) I_{m}+\left(\frac{\beta_{m} S_{m}}{\mu_{m} N_{H}}-\frac{m_{1}}{R_{0}}\right)\left(\left(I_{H 1}+I_{H 2}\right)\right)
$$

At the disease free equilibrium, $S_{H}=\frac{\Lambda_{H}\left[\omega_{0}+\mu_{H}(1-\rho)\right]}{\mu_{H}\left(\omega_{0}+\omega+\mu_{H}\right)}, S_{m}=\frac{\Lambda_{m}}{\mu_{m}}$ and $N_{H}=\frac{\Lambda_{H}}{\mu_{H}}$ 
Hence substituting $S_{H}, S_{m}, N_{H}$ and $m_{1}=\frac{\beta_{m} \Lambda_{m} \mu_{H}}{\Lambda_{H} \mu_{m}^{2}}$ (??) becomes;

$$
\begin{aligned}
\frac{d L}{d t} & =\left(\frac{\Lambda_{m} \beta_{H} \beta_{m} \mu_{H}\left(\mu_{H}+\gamma_{H}+k \alpha_{H}+\varepsilon\right)\left[\omega_{0}+\mu_{H}(1-\rho)\right]}{\Lambda_{H} \mu_{m}^{2} R_{0}\left(\alpha_{H}+\mu_{H}\right)\left(\gamma_{H}+\mu_{H}+\varepsilon\right)\left(\omega_{0}+\omega+\mu_{H}\right)}-1\right) I_{m} \\
& +\left(\frac{\beta_{m} \Lambda_{m} \mu_{H}}{\Lambda_{H} \mu_{m}^{2}}-\frac{\beta_{m} \Lambda_{m} \mu_{H}}{\Lambda_{H} \mu_{m}^{2} R_{0}}\right)\left(I_{H 1}-I_{H 2}\right)
\end{aligned}
$$

$$
\begin{aligned}
\frac{d L}{d t} & =\left(\frac{\Lambda_{m} \beta_{H} \beta_{m} \mu_{H}\left(\mu_{H}+\gamma_{H}+k \alpha_{H}+\varepsilon\right)\left[\omega_{0}+\mu_{H}(1-\rho)\right]}{\Lambda_{H} \mu_{m}^{2} R_{0}\left(\alpha_{H}+\mu_{H}\right)\left(\gamma_{H}+\mu_{H}+\varepsilon\right)\left(\omega_{0}+\omega+\mu_{H}\right)}-1\right) I_{m} \\
& +\left(\frac{\beta_{m} \Lambda_{m} \mu_{H}}{\Lambda_{H} \mu_{m}^{2}}-\frac{\beta_{m} \Lambda_{m} \mu_{H}}{\Lambda_{H} \mu_{m}^{2} R_{0}}\right)\left(I_{H 1}-I_{H 2}\right)
\end{aligned}
$$

This implies that

$$
\begin{gathered}
\frac{d L}{d t}=\left(\frac{R_{0}^{2}}{R_{0}}-1\right) I_{m}+\left(\frac{R_{m}}{N_{H}}-\frac{R_{m}}{N_{H} R_{0}}\right)\left(I_{H 1}-I_{H 2}\right) \\
\frac{d L}{d t} \leq R_{0}\left(R_{0}-1\right) I_{m}+\frac{R_{m}}{N_{H}}\left(R_{0}-1\right)\left(I_{H 1}+I_{H 2}\right)
\end{gathered}
$$

Hence, by Lyapunov-LaSalle asymptotic stability theorem as in [10], $\mathrm{R}_{0} \leq 1$ ensures that the disease-free equilibrium state $E^{0}$ is globally asymptotically stable in $\Re_{+}^{8}$. The uniqueness of the equilibrium state $E^{0}$ in $\Re_{+}^{8}$ follows from the fact that $E^{0}$ is the only point in $\Re_{+}^{8}$ where the derivatives of both $N$ and $L$ are zero.

We have to note that $R_{0} \leq 1$ ensures that $\frac{d L}{d t}<0$ holds everywhere in $\Re_{+}^{8}$ apart from point, where $\frac{d L}{d t}=0$.

This confirms the Lyapunov-LaSalle asymptotic stability theorem, hence it means that the highest invariant set of the system, is globally asymptotically stable.

By the global stability of the disease-free equilibrium state, we mean that for any initial rate of infection, dengueinfection will graduallygo into extinction from the population in the long run, if $R_{0} \leq 1$ holds. This therefore, provides the condition for the migration rate $q_{2}$ that is necessary for eradication of the dengue infection.

$R_{0}>1$ implies that one infected individual that comes into an entirely susceptible human population will cause an average of more than one infected in the next generation; hence, in this case there may be an outbreak of dengue infection in such population and the infection will persist. Standard linear analysis reveals that when the basic reproduction rate $R_{0}$ is greater than 1 , then DFE is unstable. But a supercritical bifurcation occurs at $R_{0}=1$, and a stable positive equilibrium appears.

\subsubsection{Global stability of the endemic equilibrium}

In this section, we studied the stability of the endemic equilibrium of model (3.1) using a special case where vaccine does not wane $\left(\omega_{0}=0\right)$, no continous vaccination $(\omega=0)$, but there is cohort vaccination of newlyrecruited susceptible individuals $(\rho \neq 0)$ and the associated disease-induced mortality is negligible $(\varepsilon=0)$ 
Therefore substituting $\omega_{0}=\omega=\varepsilon=0$ into system (??) we obtain,

$$
\begin{aligned}
\frac{d V_{H}}{d t} & =\Lambda_{H} \rho+q_{3} M-\mu_{H} V_{H} \\
\frac{d S_{H}}{d t} & =(1-\rho) \Lambda_{H}-\frac{\beta_{H} I_{m} S_{H}}{N_{H}}-\mu_{H} S_{H} \\
\frac{d I_{H 1}}{d t} & =\frac{\beta_{H} I_{m} S_{H}}{N_{H}}+q_{1} M-\left(\alpha_{H}+\mu_{H}\right) I_{H 1} \\
\frac{d I_{H 2}}{d t} & =q_{2} M+k \alpha_{H} I_{H 1}-\left(\gamma_{H}+\mu_{H}+\varepsilon\right) I_{H 2} \\
\frac{d M}{d t} & =-\left(q_{1}+q_{2}+q_{3}+\mu_{H}\right) M \\
\frac{d R_{H}}{d t} & =\gamma_{H} I_{H 2}+(1-k) \alpha_{H} I_{H 1}-\mu_{H} R_{H} \\
\frac{d S_{m}}{d t} & =\Lambda_{m}-\frac{\beta_{m}\left(I_{H 1}+I_{H 2}\right) S_{m}}{N_{m}}-\mu_{m} S_{m} \\
\frac{d I_{m}}{d t} & =\frac{\beta_{m}\left(I_{H 1}+I_{H 2}\right) S_{m}}{N_{m}}-\mu_{m} I_{m}
\end{aligned}
$$

It is important to noted that setting $\varepsilon=0$ in system (3.9) implies that $N_{H} \rightarrow \frac{\Lambda_{H}}{\mu_{H}}$ as $t \rightarrow \infty$. Using $N_{H}=\frac{\Lambda_{H}}{\mu_{H}}$ in (3.9) gives

$$
\begin{gathered}
\frac{\beta_{H}}{N_{H}}=\frac{\beta_{H}}{\Lambda_{H}}=\beta_{1} \text { also } \\
\frac{\beta_{m}}{N_{m}}=\frac{\beta_{m} \mu_{m}}{\Lambda_{m}}=\beta_{2}
\end{gathered}
$$

The basic reproduction number of the reduced model (3.9) is given by

$$
\begin{gathered}
R_{0 r}=\sqrt{\frac{\beta_{1} \beta_{2} \Lambda_{m}^{2}(1-\rho)\left(\mu_{H}+\kappa \alpha_{H}+\gamma_{H}\right)}{\mu_{m}^{3}\left(\alpha_{H}+\mu_{H}\right)\left(\mu_{H}+\gamma_{H}\right)}} \\
R_{0 r}^{2}=\frac{\beta_{1} \beta_{2} \Lambda_{m}^{2}(1-\rho)\left(\mu_{H}+\kappa \alpha_{H}+\gamma_{H}\right)}{\mu_{m}^{3}\left(\alpha_{H}+\mu_{H}\right)\left(\mu_{H}+\gamma_{H}\right)}
\end{gathered}
$$

First we show that there exist endemic equilibria (that is, equilibria where the infected compartments are non-zero) of the reduced model (3.9).

Setting $\lambda_{1}=\beta_{1} I_{m}, \Lambda_{1}=\rho \Lambda_{H}, \Lambda_{2}=(1-\rho) \Lambda_{H}$ and $\lambda_{1}=\beta_{2}\left(I_{H 1}+I_{H 2}\right)$, then substituting, we have

$$
\begin{aligned}
\frac{d V_{H}}{d t} & =\Lambda_{1}+q_{3} M-\mu_{H} V_{H} \\
\frac{d S_{H}}{d t} & =\Lambda_{2}-\lambda_{1} S_{H}-\mu_{H} S_{H} \\
\frac{d I_{H 1}}{d t} & =\lambda_{1} S_{H}+q_{1} M-\left(\alpha_{H}+\mu_{H}\right) I_{H 1} \\
\frac{d I_{H 2}}{d t} & =q_{2} M+k \alpha_{H} I_{H 1}-\left(\gamma_{H}+\mu_{H}\right) I_{H 2} \\
\frac{d M}{d t} & =-\left(q_{1}+q_{2}+q_{3}+\mu_{H}\right) M \\
\frac{d R_{H}}{d t} & =\gamma_{H} I_{H 2}+(1-k) \alpha_{H} I_{H 1}-\mu_{H} R_{H} \\
\frac{d S_{m}}{d t} & =\Lambda_{m}-\lambda_{2} S_{m}-\mu_{m} S_{m} \\
\frac{d I_{m}}{d t} & =\lambda_{2} S_{m}-\mu_{m} I_{m}
\end{aligned}
$$




$$
\begin{aligned}
& V_{H}^{* *}=\frac{\Lambda_{1}}{\mu_{H}} \\
& S_{H}^{* * *}=\frac{\Lambda_{2}}{\lambda_{1}^{* *}+\mu_{H}} \\
& I_{H 1}^{* *}=\frac{\lambda_{1}^{* *} S_{H}^{* *}}{\alpha_{H}+\mu_{H}} \\
& I_{H 2}=\frac{k \alpha_{H} I_{H 1}^{* * 1}}{\gamma_{H}+\mu_{H}} \\
& M^{* *}=0 \\
& R_{H}^{* *}=\frac{\gamma_{H} I_{H 2}^{* *}+(1-k) \alpha_{H} I_{H 1}^{* *}}{\mu_{H}} \\
& S_{m}^{* *}=\frac{\Lambda_{m}}{\lambda_{m}^{* *}+\mu_{m}} \\
& I_{m}^{* *}=\frac{\lambda_{2}^{* *} S_{m}^{* *}}{\mu_{m}}
\end{aligned}
$$

Where $\lambda_{1}^{* *}=\beta_{1} I_{m}^{* *}$ and $\lambda_{2}^{* *}=\beta_{2}\left(I_{H 1}^{* *}+I_{H 2}^{* *}\right)$

By substituting (3.11) into $\lambda_{1}^{* *}$ and $\lambda_{2}^{* *}$, we have;

$$
\begin{aligned}
& \qquad \begin{array}{l}
a_{0}\left(\lambda^{* *}\right)^{2}+a_{1}\left(\lambda^{* *}\right)=0 \\
\text { where } a_{0}=\mu_{m}^{2}+\mu_{m} k_{1} k_{2} \text { and } a_{1}=\mu_{m}^{2} \mu_{H}-\beta_{1} \Lambda_{m} k_{1} k_{2} \quad k_{1}=\frac{\beta_{2} \Lambda_{2}}{\left(\alpha_{H}+\mu_{H}\right)\left(\gamma_{H}+\mu_{H}\right)} \\
k_{2}=\gamma_{H}+\mu_{H}+\kappa \alpha_{H}
\end{array}
\end{aligned}
$$

The endemic equilibria of the reduced model (3.11) can be obtained by solving for $\lambda_{1}^{* *}$ in (??) and substituting the positive values into (3.9).

Lemma 3.3: The reduced model (3.10) has a unique positive endemic equilibrium if $a_{1}<0$, whenever $R_{o r}>1$.

Theorem 3.4: The unique endemic equilibrium of the reduced model (3.10) is globally asymptotical stable if $R_{0 r}>1$.

Proof: consider the non-linear Lyapunov function for the reduced model (3.10);

$$
\begin{aligned}
& L=c_{1}\left[S_{H}-S_{H}^{* *}-S_{H}^{* *} \operatorname{In}\left(\frac{S_{H}}{S_{H}^{* *}}\right)+I_{H 1}-I_{H 1}^{* *}-I_{H 1}^{* *} \operatorname{In}\left(\frac{I_{H 1}}{I_{H 1}^{* *}}\right)\right]+c_{2}\left[I_{H 2}-I_{H 2}^{* *}-I_{H 2}^{* *} \operatorname{In}\left(\frac{I_{H 2}}{I_{H 2}^{* *}}\right)\right] \\
& +c_{3}\left[M-M^{* *}-M^{* *} \operatorname{In}\left(\frac{M}{M^{* *}}\right)\right]+c_{4}\left[S_{m}-S_{m}^{* *}-S_{m}^{* *} \operatorname{In}\left(\frac{S_{m}}{S_{m}^{* *}}\right)+I_{m}-I_{m}^{* *}-I_{m}^{* *} \operatorname{In}\left(\frac{I_{m}}{I_{m}^{* *}}\right)\right]
\end{aligned}
$$

with Lyapunov derivative

$$
\begin{aligned}
\frac{d L}{d t}= & c_{1}\left[\left(1-\frac{S_{H}^{* *}}{S_{H}}\right)\left\{\Lambda_{2}-\beta_{1} I_{m} S_{H}-\mu_{H} S_{H}\right\}+\left(1-\frac{I_{H 1}^{* *}}{I_{H 1}}\right)\left\{\beta_{1} I_{m} S_{H}+q_{1} M-\left(\alpha_{H}+\mu_{H}\right) I_{H 1}\right\}\right]+ \\
& c_{2}\left[\left(1-\frac{I_{H 2}^{* *}}{I_{H 2}}\right)\left\{q_{2} M+k \alpha_{H} I_{H 1}-\left(\gamma_{H}+\mu_{H}\right) I_{H 2}\right\}\right]+c_{3}\left[\left(1-\frac{M^{* *}}{M}\right)\left\{-\left(q_{1}+q_{2}+q_{3}+\mu_{H}\right) M\right\}\right]+ \\
& c_{4}\left[\left(1-\frac{S_{m}^{* *}}{S_{m}}\right)\left\{\Lambda_{m}-\beta_{2}\left(I_{H 1}+I_{H 2}\right) S_{m}-S_{m}\right\}+\left(1-\frac{I_{m}^{* *}}{I_{m}}\right)\left\{\beta_{2}\left(I_{H 1}+I_{H 2}\right) S_{m}-\mu_{m} I_{m}\right\}\right]
\end{aligned}
$$


At the steady state;

$$
\begin{aligned}
& \Lambda_{2}=\beta_{1} I_{m}^{* *} S_{H}^{* *}+\mu_{H} S_{H}^{* *} \\
& M^{* *}=0 \\
& \Lambda_{m}=\beta_{2}\left(I_{H 1}^{* *}+I_{H 2}^{* *}\right) S_{m}^{* *}+\mu_{m} S_{m}^{* *}
\end{aligned}
$$

Hence (3.11) becomes;

(3.12)

$$
\begin{aligned}
\frac{d L}{d t} & =c_{1}\left[\left(1-\frac{S_{H}^{* *}}{S_{H}}\right)\left\{\beta_{1} I_{m}^{* *} S_{H}^{* *}+\mu_{H} S_{H}^{* *}-\beta_{1} I_{m} S_{H}-\mu_{H} S_{H}\right\}\left(1-\frac{I_{H 1}^{* *}}{I_{H 1}}\right)\left\{\beta_{1} I_{m} S_{H}+q_{1} M-\left(\alpha_{H}+\mu_{H}\right) I_{H 1}\right\}\right] \\
& +c_{2}\left[\left(1-\frac{I_{H 2}^{* *}}{I_{H 2}}\right)\left\{q_{2} M+k \alpha_{H} I_{H 1}-\left(\gamma_{H}+\mu_{H}\right) I_{H 2}\right\}\right] \\
& +c_{3}\left[\left\{-\left(q_{1}+q_{2}+q_{3}+\mu_{H}\right) M\right\}\right] \\
& +c_{4}\left[\left(1-\frac{S_{m}^{* *}}{S_{m}}\right)\left\{\beta_{2}\left(I_{H 1}^{* *}+I_{H 2}^{* *}\right) S_{m}^{* *}+\mu_{m} S_{m}^{* *}-\beta_{2}\left(I_{H 1}+I_{H 2}\right) S_{m}-\mu_{m} S_{m}\right\}+\left(1-\frac{I_{m}^{* *}}{I_{m}}\right)\left\{\beta_{2}\left(I_{H 1}+I_{H 2}\right) S_{m}-\mu_{m} I_{m}\right\}\right]
\end{aligned}
$$

After rigorous computation we have;

$$
\begin{aligned}
& c_{1}=k_{1} \beta_{2} S_{m}^{* *} \\
& c_{2}=\beta_{2} S_{m}^{* *} \\
& c_{3}=k_{2} \beta_{2} S_{m}^{* *} \\
& c_{4}=\gamma_{H}
\end{aligned}
$$

where

$$
\begin{aligned}
& k_{1}=\frac{\kappa \alpha_{H}+\gamma_{H}}{\alpha_{H}} \\
& k_{2}=\frac{q_{1}\left(\kappa \alpha_{H}+\gamma_{H}\right)+q_{2} \alpha_{H}}{\alpha_{H}\left(q_{1}+q_{2}+q_{3}\right)}
\end{aligned}
$$

Therefore (3.12) becomes;

(3.13)

$$
\begin{aligned}
& \frac{d L}{d t}=k_{1} \beta_{2} S_{m}^{* *}\left[\left(1-\frac{S_{H}^{* *}}{S_{H}}\right)\left\{\beta_{1} I_{m}^{* *} S_{H}^{* *}+\mu_{H} S_{H}^{* *}-\beta_{1} I_{m} S_{H}-\mu_{H} S_{H}\right\}\left(1-\frac{I_{H 1}^{* *}}{I_{H 1}}\right)\left\{\beta_{1} I_{m} S_{H}+q_{1} M-\left(\alpha_{H}+\mu_{H}\right) I_{H 1}\right\}\right] \\
& +\beta_{2} S_{m}^{* *}\left[\left(1-\frac{I_{H 2}^{* *}}{I_{H 2}}\right)\left\{q_{2} M+k \alpha_{H} I_{H 1}-\left(\gamma_{H}+\mu_{H}\right) I_{H 2}\right\}\right]+k_{2} \beta_{2} S_{m}^{* *}\left[\left\{-\left(q_{1}+q_{2}+q_{3}+\mu_{H}\right) M\right\}\right] \\
& +\gamma_{H}\left[\left(1-\frac{S_{m}^{* *}}{S_{m}}\right)\left\{\beta_{2}\left(I_{H 1}^{* *}+I_{H 2}^{* *}\right) S_{m}^{* *}+\mu_{m} S_{m}^{* *}-\beta_{2}\left(I_{H 1}+I_{H 2}\right) S_{m}-\mu_{m} S_{m}\right\}+\left(1-\frac{I_{m}^{* *}}{I_{m}}\right)\left\{\beta_{2}\left(I_{H 1}+I_{H 2}\right) S_{m}-\mu_{m} I_{m}\right\}\right] \\
& \begin{aligned}
\frac{d L}{d t}= & k_{1} \beta_{2} S_{m}^{* *}\left[\beta_{1} I_{m}^{* *} S_{H}^{* *}-\frac{\beta_{1} I_{m}^{* *} S_{H}^{* * 2}}{S_{H}}+\mu_{H} S_{H}^{* *}-\frac{\mu_{H} S_{H}^{* * 2}}{S_{m}}-\mu_{H} S_{H}+\mu_{m} S_{H}^{* *}\right]+k_{1} \beta_{2} S_{m}^{* *}\left[\mu_{H} I_{H 1}^{* *}-\mu_{H} I_{H 1}\right] \\
+ & \beta_{2} S_{m}^{* *}\left[\mu_{H} I_{H 2}^{* *}-\mu_{H} I_{H 2}\right]+\gamma_{H}\left[\beta_{2}\left(I_{H 1}^{* *}+I_{H 2}^{* *}\right) S_{m}^{* *}-\frac{\beta_{2}\left(I_{H 1}^{* *}+I_{H 2}^{* *}\right) S_{m}^{* *}}{S_{m}}+\mu_{m} S_{m}^{* *}-\frac{\mu_{m} S_{m}^{* *}}{S_{m}}-\mu_{m} S_{m}+\mu_{m} S_{m}^{* *}\right] \\
+ & \gamma_{H}\left[\mu_{m} I_{m}^{* *}-\mu_{m} I_{m}\right] \\
(3.14) \quad & \frac{d L}{d t}=k_{1} \beta_{1} \beta_{2} S_{m}^{* *} I_{m}^{* *} S_{H}^{* *}\left[1-\frac{S_{H}^{* *}}{S_{H}}\right]+\mu_{H} k_{1} \beta_{2} S_{H}^{* *} S_{m}^{* *}\left[2-\frac{S_{H}^{* *}}{S_{H}}-\frac{S_{H}}{S_{H}^{* *}}\right]+\mu_{H} k_{1} \beta_{2} S_{m}^{* *} I_{H 1}^{* *}\left[1-\frac{I_{H 1}}{I_{H 1}^{* *}}\right] \\
+ & \mu_{H} \beta_{2} S_{m}^{* *} I_{H 2}^{* *}\left[1-\frac{I_{H 2}}{I_{H 2}^{* *}}\right]+\gamma_{H} \beta_{2}\left(I_{H 1}^{* *}+I_{H 2}^{* *}\right) S_{m}^{* *}\left[1-\frac{S_{m}^{* *}}{S_{m}}\right]+\gamma_{H} \mu_{m} S_{m}^{* *}\left[2-\frac{S_{m}^{* *}}{S_{m}}-\frac{S_{m}}{S_{m}^{* *}}\right] \\
+ & \gamma_{H} \mu_{m} I_{m}^{* *}\left[1-\frac{I_{m}}{I_{m}^{* *}}\right]
\end{aligned} \\
& (3.15) \quad
\end{aligned}
$$


Finally, since the arithmetic mean is greater geometric mean, then it follows that. Again, since all the model parameters are non-negative, it follows that $\frac{d L}{d t} \leq 0$. for $R_{0 r}>1$.

Hence $L$ is a Lyapunov function of the reduced model. Therefore, it follows from [10] that

$$
\begin{aligned}
& \lim _{t \rightarrow \infty} S_{H}(t)=S_{H}^{* *} \\
& \lim _{t \rightarrow \infty} V_{H}(t)=V_{H}^{* *} \\
& \lim _{t \rightarrow \infty} I_{H 1}(t)=I_{H 1}^{* *} \\
& \lim _{t \rightarrow \infty} I_{H 2}(t)=I_{H 2}^{* *} \\
& \lim _{t \rightarrow \infty} M(t)=M^{* *} \\
& \lim _{t \rightarrow \infty} R_{H}(t)=R_{H}^{* *} \\
& \lim _{t \rightarrow \infty} S_{m}(t)=S_{m}^{* *} \\
& \lim _{t \rightarrow \infty} I_{m}(t)=I_{m}^{* *}
\end{aligned}
$$

Thus it means that for the special case $\omega_{0}=\omega=\varepsilon=0$, the Dengue fever will persist in the population whenever the associated reproduction number exceeds unity ( that is, for $R_{0 r}>1$.)

\subsection{Numerical Simulation;}

The transmission of dengue disease in this study is based on the SIR model with vaccination and migration effect. The numerical simulations were done using the following values of parameters; 


\begin{tabular}{|c|c|c|c|}
\hline Symbols & Description & Value( per day) & Source \\
\hline$V_{H}$ & Number of Vaccinated individual at time $t$ & 100 & Variable \\
\hline$S_{H}$ & The number of susceptible human at time $t$ & 80 & Variable \\
\hline$I_{H 1}$ & The number of common dengue individuals at time $t$ & 40 & Variable \\
\hline$I_{H 2}$ & The number of severe infected individuals at time $t$ & 40 & Variable \\
\hline$M$ & The number of immigrants at time $t$ & 85 & Variable \\
\hline$R_{H}$ & The number of recovered human individual at time $t$ & 100 & Variable \\
\hline$S_{m}$ & Number of susceptible mosquito at time $t$ & 75 & Variable \\
\hline$I_{m}$ & Number of infected mosquito at time $t$ & 50 & Variable \\
\hline$\Lambda_{H}$ & Recruitment level of human & 0.405 & {$[9]$} \\
\hline$\Lambda_{m}$ & Recruitment level of mosquito & 110 & {$[9]$} \\
\hline$\Lambda_{M}$ & Recruitment level of migrated class & 0.5 & Estimated \\
\hline$\beta_{H}$ & Contact rate of human & 0.75 & {$[15]$} \\
\hline$\beta_{m}$ & Contact rate of mosquito & 0.000035 & {$[9]$} \\
\hline$\alpha_{H}$ & The rate at which the infected individuals become recovered & 0.012 & {$[9]$} \\
\hline$\kappa$ & Fraction of individual who become severely infected & 0.06 & Estimated \\
\hline$\gamma_{H}$ & The rate at which severe infected individuals are recovering & 0.012 & {$[9]$} \\
\hline$\omega$ & The rate at which susceptible individuals are vaccinated & 0.75 & {$[5]$} \\
\hline$\omega_{0}$ & Vaccine weaning rate & 0.2 & Estimated \\
\hline$q_{1}$ & The rate of flow from Migrated class to infected class & 0.2 & Estimated \\
\hline$q_{2}$ & The rate of flow from Migrated class to severe infected class & 0.1 & Estimated \\
\hline$q_{3}$ & The rate at which immigrants are vaccinated & 0.3 & Estimated \\
\hline$q_{4}$ & The rate at which immigrants become susceptible & 0.4 & Estimated \\
\hline$\mu_{H}$ & Natural death rate of human & 0.000045 & {$[9]$} \\
\hline$\mu_{m}$ & Natural death rate of mosquito & 0.05 & {$[9]$} \\
\hline$\rho$ & Fraction of recruited individual who are vaccinated & 0.75 & {$[5]$} \\
\hline$\varepsilon$ & Disease induced death rate & 0.04 & {$[3]$} \\
\hline$\eta$ & Level of infectiousness of the severe infected class & 1.5 & $\eta \geq 1$ \\
\hline
\end{tabular}




\section{Results ANd Discussion}

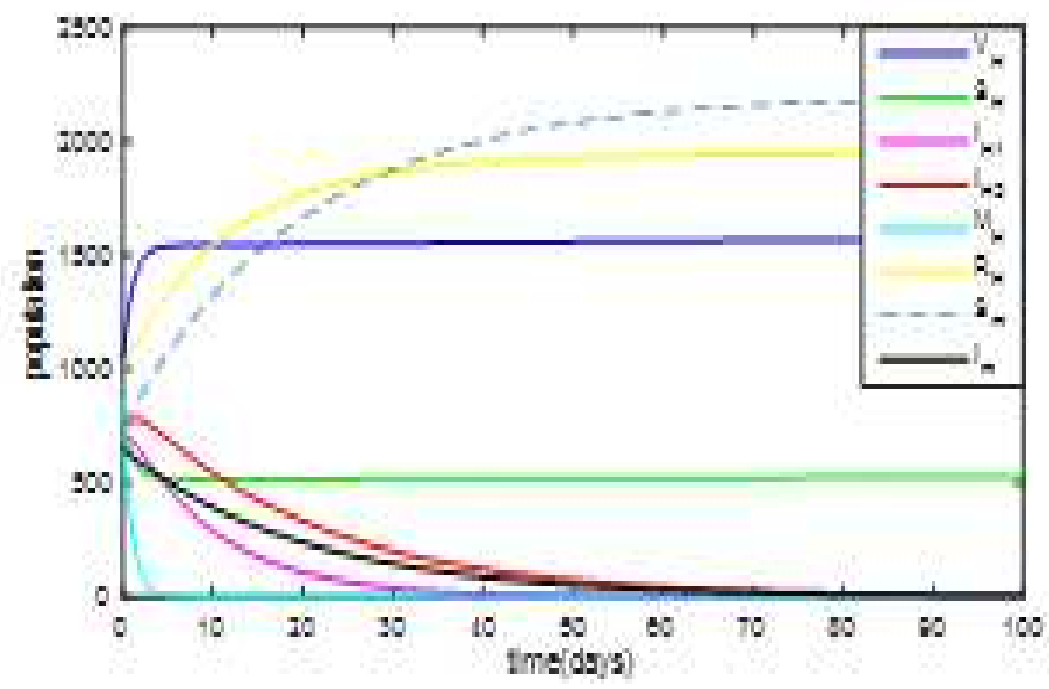

Fig.1: Plot of the population against time

From the figure above, we observe that all the disease compartments $\mathrm{I}_{H 1}, \mathrm{I}_{H 2}$, and $\mathrm{M}_{H}$ are all reduced. This implies that the spread of dengue infection over a period can be minimized, when proper measures like vaccination of susceptible population and mounting a proper check on the immigrants. Our simulation also reveals that the susceptible mosquitos' remain in the population, when the susceptible mosquitoes bite any of the infected classes, this will result to further spread of dengue infection. Therefore, one of the ways of controlling dengue infection is by controlling the breed of mosquitoes and avoiding bites of mosquitoes.

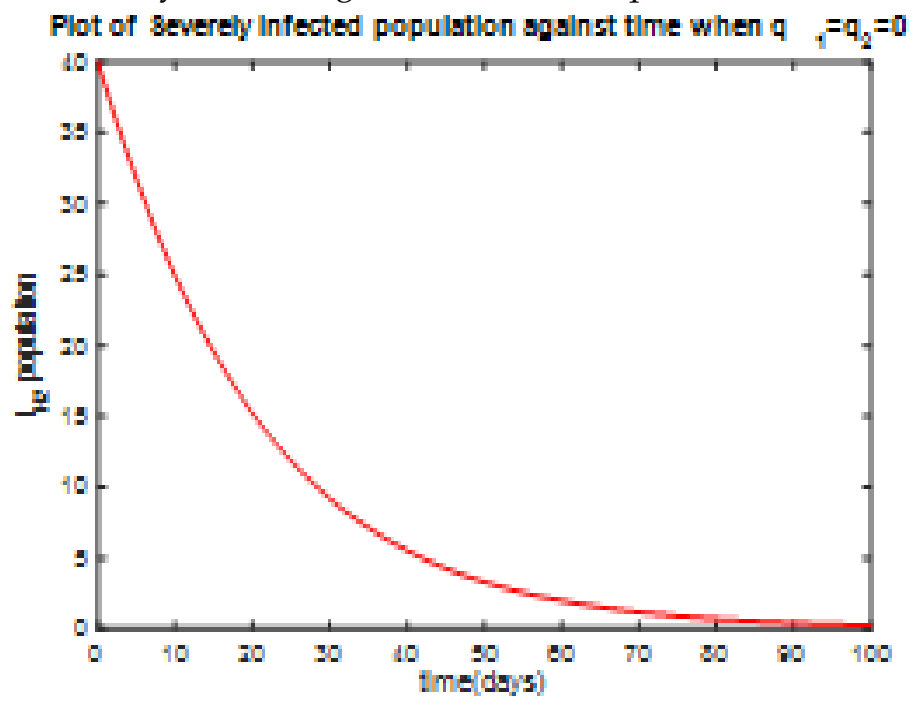

Fig. 2: Plot of severe infected population against time

Here, we studied the behavior of the severely infected individual when immigrants are neither infected nor severely infected. Our simulation reveals that the population will be reduced over time. Precisely for a period of 555days the entire severely infected population will go into extinction, when $\mathrm{q}_{1}=\mathrm{q}_{2}=0$. 


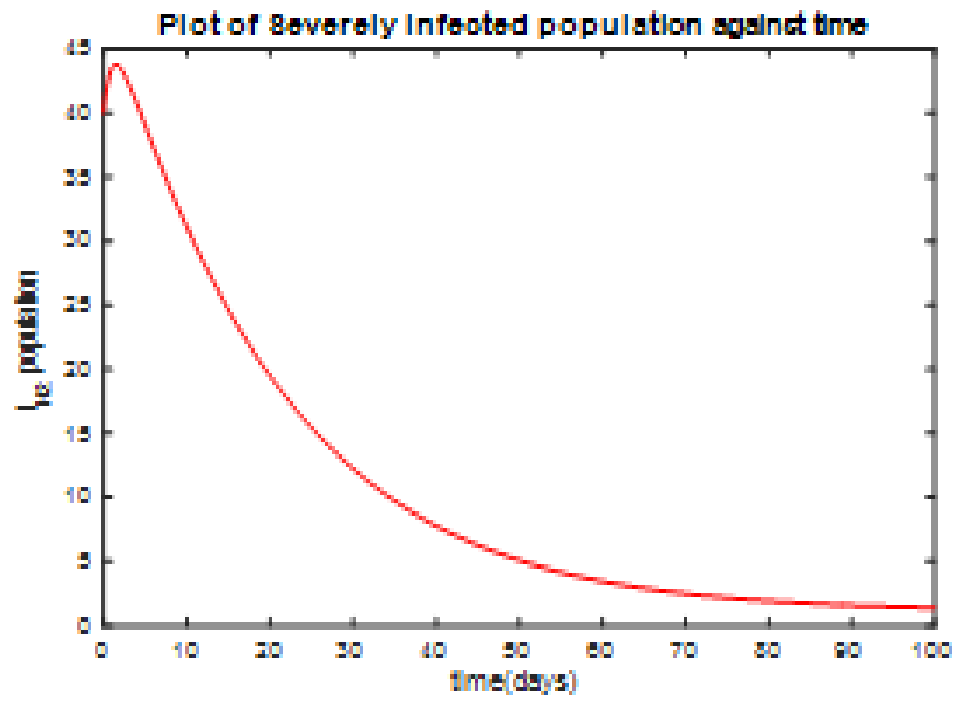

Fig.3: Plot of severely infected against time

Also, we look at the severely infected population over time when immigrants are either severely infected or infected, (that is, we studied the reverse case of Fig.2). Our simulation shows that with vaccination alone the severely infected population can be reduced but cannot be entirely wiped in the long run, since it remains constant after about 93days

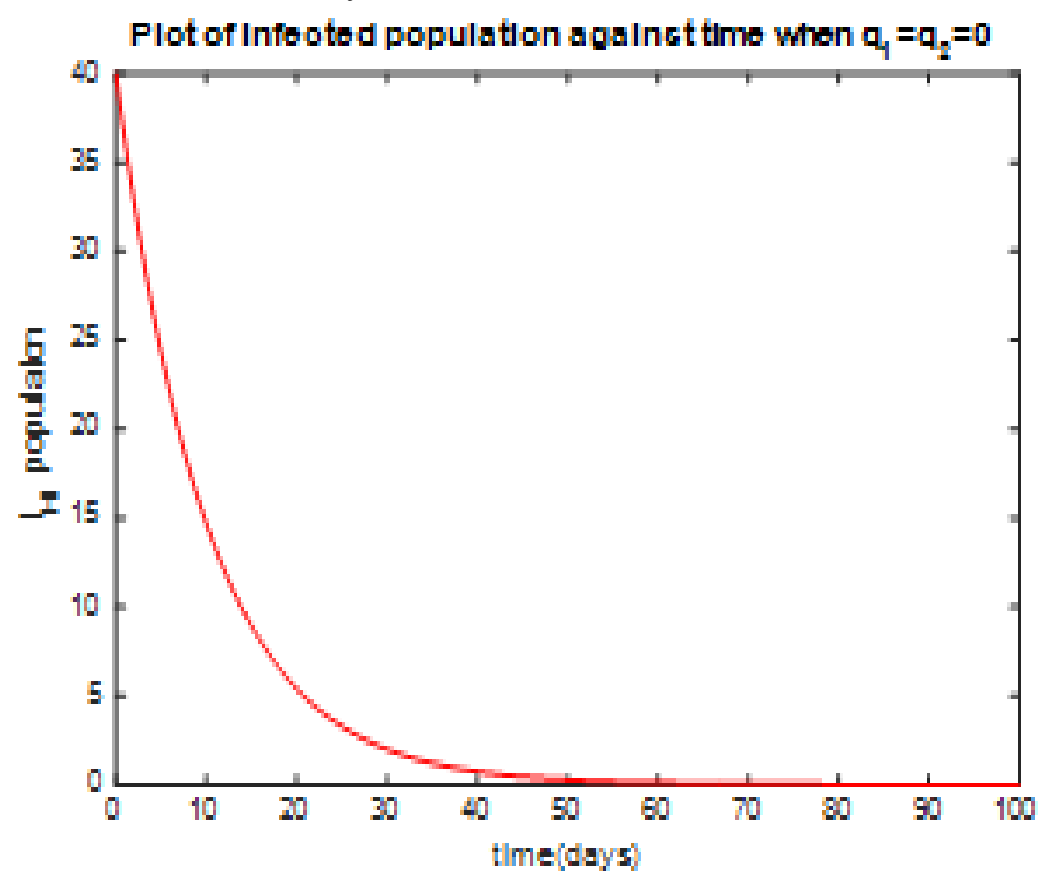

Fig.4: Plot of infected population against time

Again we looked into the infected population when immigrants are neither infected nor severely infected. Our simulation reveals that the infected population will fizzle out just in 53days when $\mathrm{q}_{1}=\mathrm{q}_{2}=0$. 


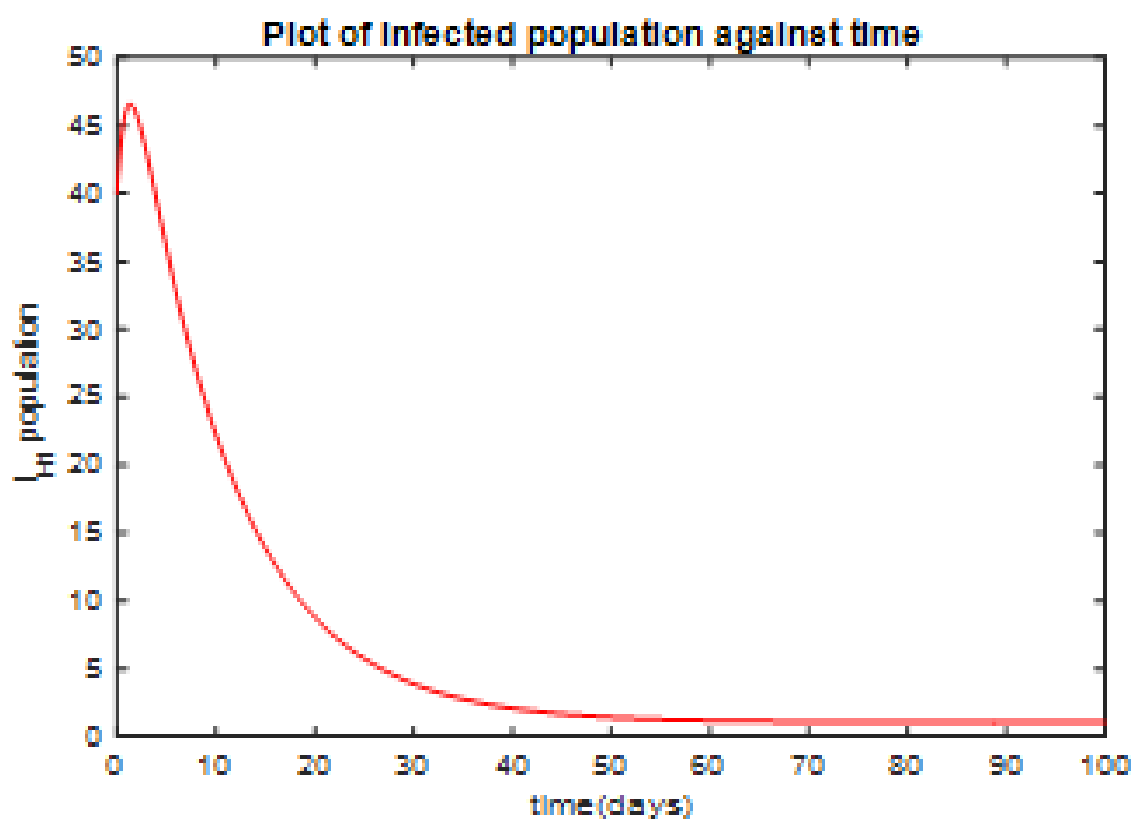

Fig.5: Plot of infected population against time

Also, we look at the infected population over time when immigrants are either severely infected or infected, (that is, we studied the reverse case of fig. 4). Our simulation shows that with vaccination alone the infected population can be reduced but cannot be entirely wiped in the long run, since it remains constant after about 55days.

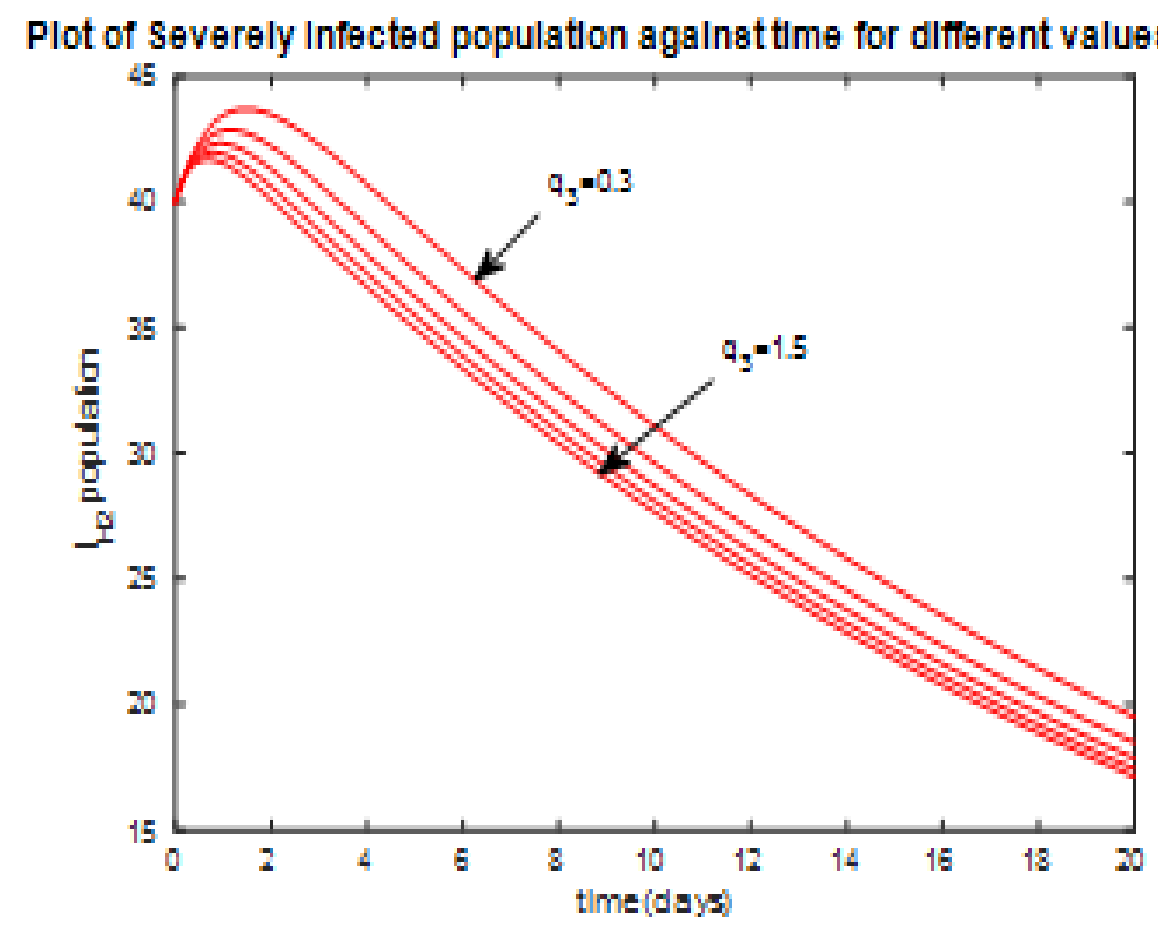

Fig.6: Plot of Severely infected population against time

Here, we studied the effect of vaccinating the immigrants, from our simulation it is clear that increase in the vaccination rate of immigrants contributes to a reduced population of the severely infected individuals. 


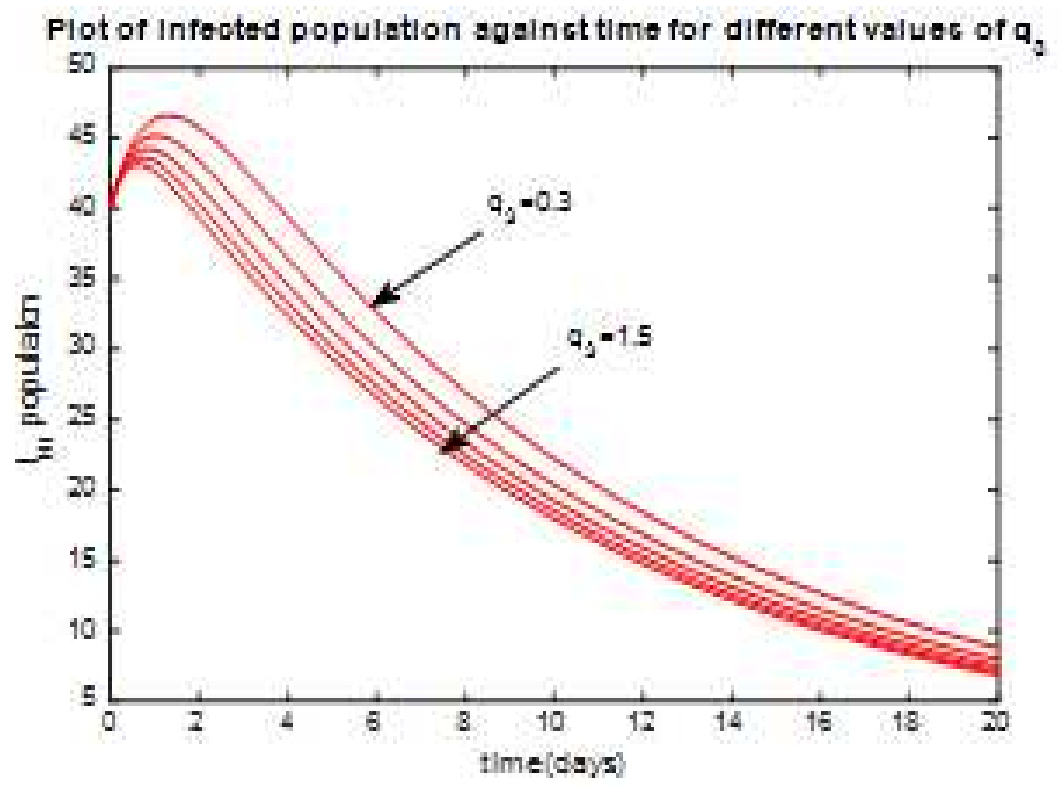

Fig.7: Plot ofinfected population against time

Again, we studied the effect of vaccinating the immigrants, from our simulation it is clear that increase in the vaccination rate of immigrants contributes to a reduced population of the infected individuals.

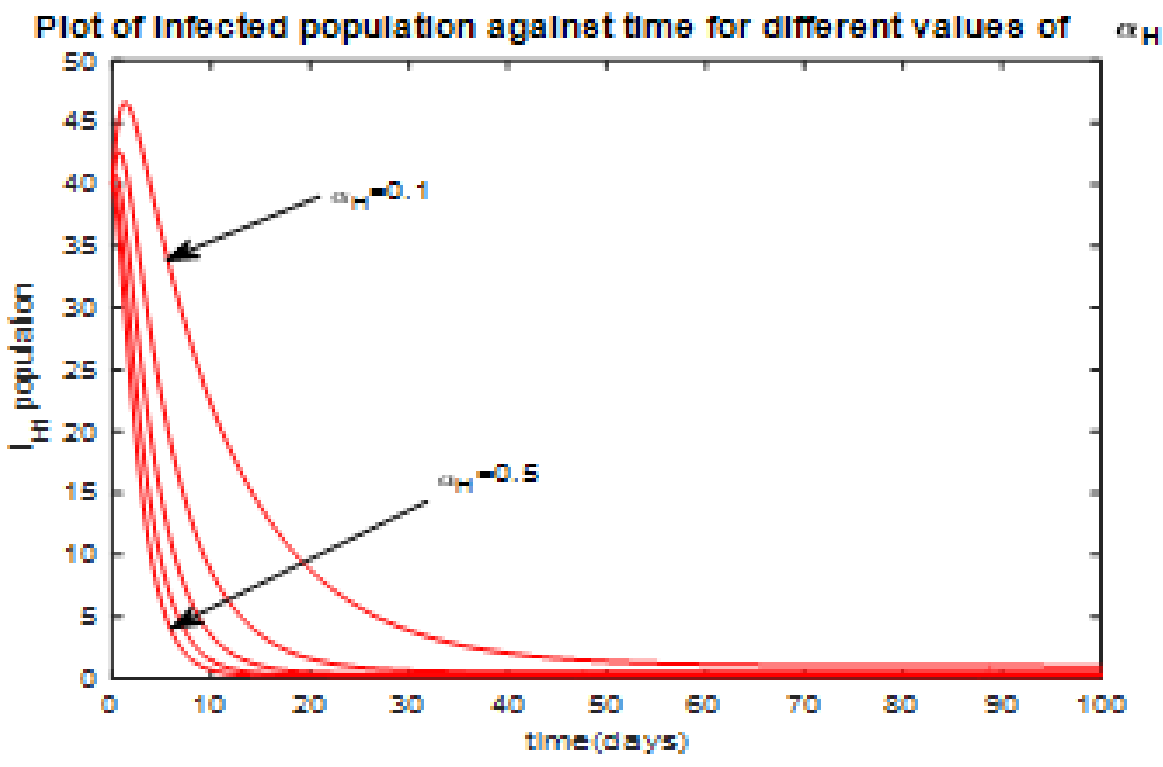

Fig.8: Plot ofinfected population against time

In this simulation, we studied the effect of recovery rate on the infected population. Our result shows that if the recovery rate is increasing, then less individuals are infected and the tendency of individual becoming severely infected is reduced. 


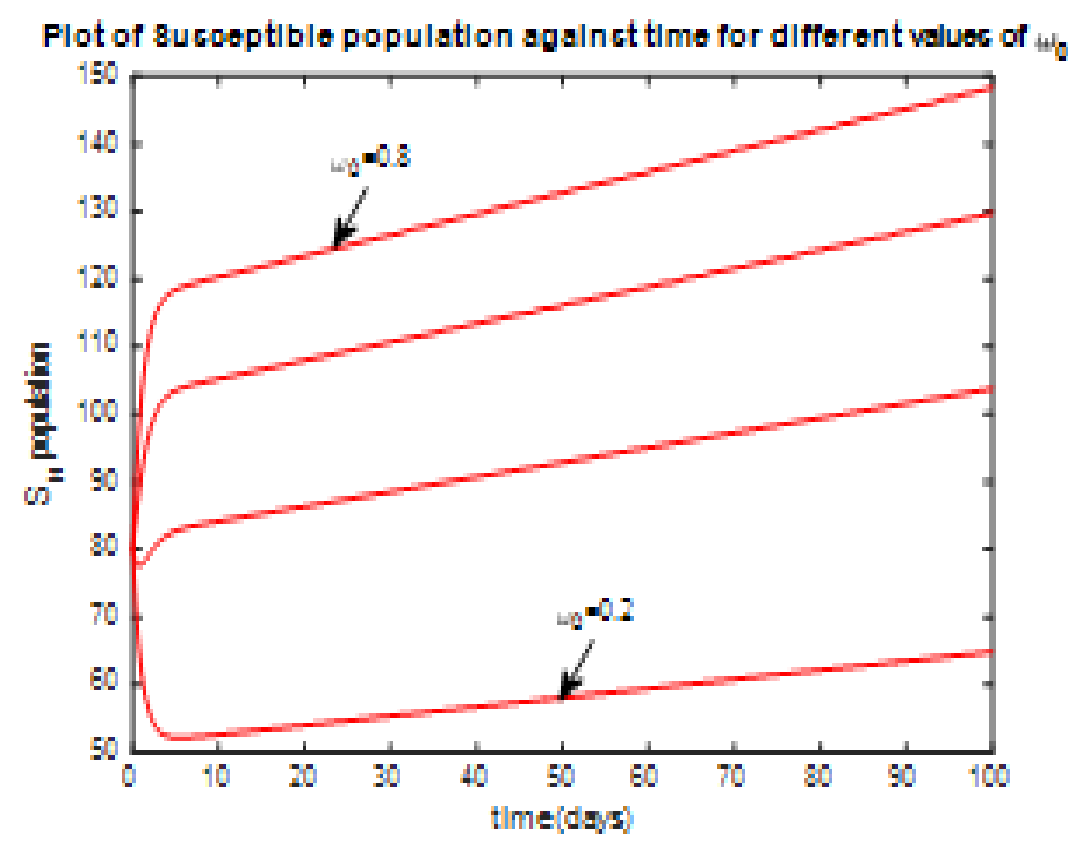

Fig.9: Plot ofinfected population against time

The simulation shows that if there increase in the rate at which vaccination weans then, the number of susceptible individual will increase.

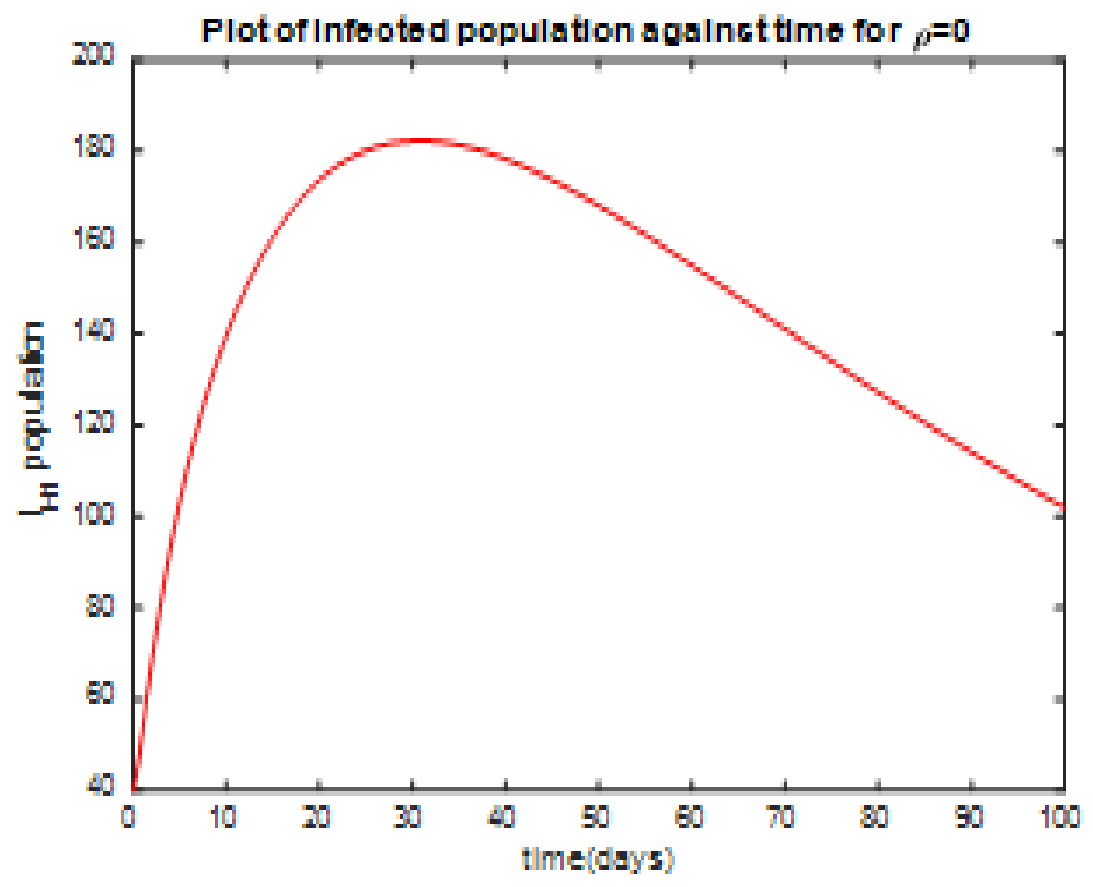

Fig.10: Plot ofinfected population against time " $R_{0}>1$

In this simulation, we analyze the spread of dengue infection without vaccination impact, but there is a proper check on the immigrants, they are neither infected nor severely infected. Our simulation shows that, in the long run, a good number of the population will still be infected with dengue disease. This suggests that only check on the immigrants cannot prevent the spread of dengue infection. But also what can be done to ensure that they are prevented from been infected should be put in place. 


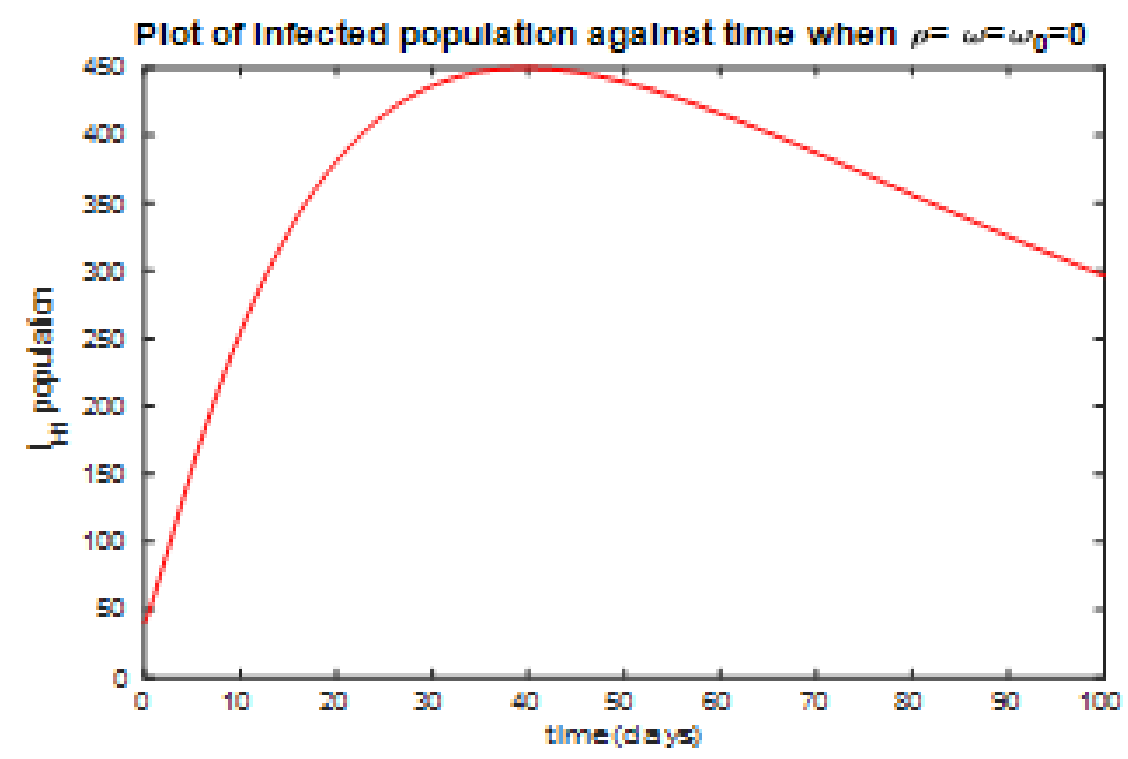

Fig.11: Plot ofinfected population against time " $R_{0}>1$ "

Here, we studied the infected population without continuous influence of dengue vaccination campaign and without check on the immigrants; here we observe that there is a tremendous increase in the number of infected individual.

It is important to emphasis at this point that vaccination alone or check on the immigrants alone can prevent the spread of dengue infection as suggest by our simulation (fig.1-5) and 10. Rather both measures must be considered to effectively fight against the spread of dengue infection.

\section{Summary, Conclusion and Recommendation}

5.1. Summary. Dengue disease is one of thevery prevalent diseases worldwide and spreading in new areas. Therefore, there is an urgent need to develop models that will effectively bring the threat posed by dengue disease to stop.

In this present study, we have used SIR-SI epidemic model to study the in?uence of dengue vaccination campaignand migrationeffect in transmission dynamics of dengue disease. There is no effective treatment of dengue disease. So, we introduced check and vaccination of immigrants as a measure in the model which can help in reducing the burden of dengue disease.

5.2. Conclusion. Basic reproduction number is a powerful parameter, which determines whether the disease comes under control or becomes more prevalent. Results in this present work show that DFE of the model is locally and globally stable when $R_{0}<1$; unstable and endemic equilibrium point exists when $R_{0}>1$. Our simulation shows that for higher values of vaccination rate and reduced immigration, the basic reproduction number is reduced, thereby contributing to the local and global stability of our model. Also for a reduced vaccination and unchecked immigration, our model becomes unstable and hence out of control.

5.3. Recommendations. : The following recommendations were made:

(1) Vaccination strategy cannot be the only control for dengue disease

(2) Checks on immigrants also cannot control the spread of dengue 
(3) To reduce the burden of dengue, there must be a continuous vaccination of not only the susceptible human population but also of immigrants.

(4) Individuals who are infected or severely infected should be restricted from entering the population or rather should be isolated from the susceptible human population, since there are still susceptible mosquitoes in the population which can still feed on both this infected immigrant and susceptible human.

(5) Both vaccination and controlled immigration can reduce the threat of dengue disease.

(6) The susceptible human population should take all possible precautions such as using mosquito repellent, mosquito net e.t.cin order to avoid the bite of mosquitoes. 


\section{REFERENCES}

[1] Aguiar, M., Stollenwerk, N and Halstead, S.B. Theimpactof the newly licensed dengue vaccine in endemic countries, PLoS Negl. Trop. Dis. 10(12) (2016), e0005179,

[2] Al-Sheikh,S.A. Modeling and analysis of an SEIR epidemic model with a limited resource for treatment. Glob. J. Sci. Front. Res. $12(14-\mathrm{F})(2012), 2249-4626$.

[3] Carvalho, S. A; da Silva, S. O. Iraziet and da Cunha Charret, Mathematical Modeling of Dengue Epidemic: Control Methods and Vaccination Strategies, Theory Biosci. 138(2)(2019), 223-239.

[4] Chan M, Johansson MA. The Incubation periods of dengue viruses; PLoS ONE, 7(2012), e50972.

[5] Chanprasopchai, P; Tang, I. M and Pongsumpun, I. M. SIR Model for Dengue Disease with Effect of Dengue Vaccination. Comput. Math. Methods Med. 2018(2018), Article ID 9861572.

[6] Chanprasopchai, P and Pongsumpun, P, The transmission dynamic of SIR modeling for dengue fever with vector infection, Proceedings of the Burapha University International Conference, Global Warming and Its impact, pp. 295-301, Pattaya, Thailand. (2014).

[7] Chanprasopchai,P.; Pongsumpun, P and.Tang, I.M. Effect of rainfall for the dynamical transmission model of the dengue disease in Thailand, Comput. Math. Methods Med. 2017(2017), Article ID 2541862.

[8] Esteva L, Vargas C. Analysis of a dengue disease transmission model, Math. Biosci. 150(1998), 131-151.

[9] Gakkhar, S. and Chavda, N. C. Impact of awareness on the spread of dengue infection in human population, Appl. Math. 4(2013), 142 147

[10] La Salle J. P. The Stability of Dynamical System; Ser. Regional Conference, Series in Applied Mathematics, SIAM. No. 25, (1976).

[11] IuriiBakach and James Braselton, A Survey of Mathematical Models of Dengue Fever; Journal of Computer Science \& Systems Biology Bakach and Braselton, J Comput. Sci. Syst. Biol. 8(2015), 5.

[12] Murray J. D. Mathematical Biology (2 ${ }^{\text {nd }}$, corrected Edition), Springer-Verlag, (2001).

[13] Phaijoo G.R and Gurung D.B. Mathematical study of dengue disease transmission in multi-patch environment. Appl. Math. 7(2016), $1521-1533$

[14] Phaijoo G. R and Gurung D.B. Mathematical model on analysis of awareness in controlling dengue disease; Int. J. Adv. Res. 4(2016), 999-1006.

[15] Pinho S. T. R; Ferreira C. P; Sterval E.; Barrreto F. R. K; Morato E.; Silva V. C and Teixeira M. G. L. Modelling the Dynamics of Dengue real epidemics; Phil. Trans. K. Sou 368(1933)(2010), Pp.5679-5693.

[16] Pongsumpun P. Transmission model for dengue disease with and without the e?ect of extrinsic incubation period. KMTL, Sci. Tech. J. $6(2006), 74-82$

[17] Pongsumpun, P. and Tang, I. M. Transmission of dengue hemorrhagic fever in an age structured population, Math. Computer Model. 37(9-10)(2003), 949-961.

[18] Recker, M., Vannice, K., Hombach, J, Jit, M and.Simmons, C. P. Assessing Dengue vaccination impact: model challenges and future directions, Vaccine, 34(38)(2016), 4461-4465.

[19] Sardar T, Rana S. Chattopadhyay. A mathematical model of dengue transmission with memory. Commun. Nonlinear Simmul. 22(1)(2015), 511-525.

[20] Shim, E. Dengue dynamics and vaccine cost-effectiveness analysis in the Philippines, Amer. J. Trop. Med. Hyg. 95(5) (2016), 1137-1147,.

[21] Side S, Noorani, MSM SEIR model for transmission of dengue fever; Int. J. Adv. Sci. Eng. Inf. Technol. 2(2012), 380-389.

[22] Soewono E, Supriatna A. K. A two - dimensional model for the transmission of dengue fever disease. Bull. Malaysian Math. Sci. Soc. 24(2001), 49-57.

[23] Van den Driessche P, Watmough J. Reproduction numbers and sub-threshold endemic equilibria for compartmental models for disease transmission. Math. Biosci. 180(2002), 2948.

[24] World Health Organization. Dengue and severe dengue, (2019)

[25] www.gooogle.com/webMD

[26] Yingyun Shen, Mathematical Models of Dengue fever and measures to control it; Ph.D Thesis, Department of Mathematics, Florida State University. (2014). 